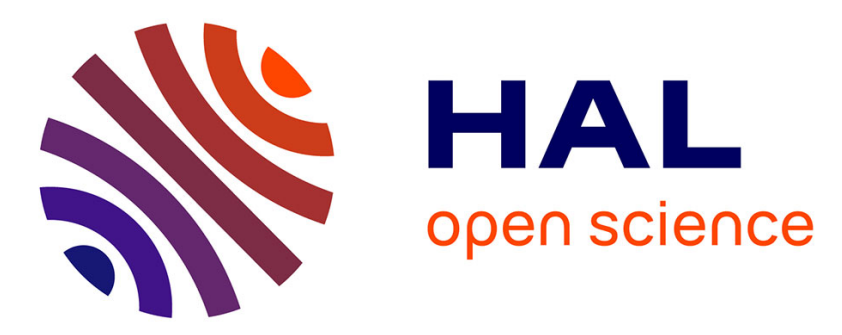

\title{
Comparaison des données rétrospectives de fécondité dans les recensements en Belgique et les enquêtes Famille en France
}

Sandra Brée, Thierry Eggerickx, Jean-Paul Sanderson, Rafael Costa

\section{- To cite this version:}

Sandra Brée, Thierry Eggerickx, Jean-Paul Sanderson, Rafael Costa. Comparaison des données rétrospectives de fécondité dans les recensements en Belgique et les enquêtes Famille en France. Population (édition française), 2016, 71 (1), 10.3917/popu.1601.0085 . halshs-01624729

HAL Id: halshs-01624729

https://shs.hal.science/halshs-01624729

Submitted on 19 Jul 2021

HAL is a multi-disciplinary open access archive for the deposit and dissemination of scientific research documents, whether they are published or not. The documents may come from teaching and research institutions in France or abroad, or from public or private research centers.
L'archive ouverte pluridisciplinaire HAL, est destinée au dépôt et à la diffusion de documents scientifiques de niveau recherche, publiés ou non, émanant des établissements d'enseignement et de recherche français ou étrangers, des laboratoires publics ou privés. 
Sandra BRÉE*, Thierry EGGERICKX*, Jean-Paul SANDERSON*, Rafael COSTA**

\title{
Comparaison des données rétrospectives de fécondité dans les recensements en Belgique et les enquêtes Famille en France
}

\begin{abstract}
Les comportements de fécondité peuvent être décrits à partir de plusieurs sources de données. Les naissances enregistrées à l'état civil donnent lieu à des bulletins statistiques qui, couplés avec les données de population issues des recensements, fournissent une estimation annuelle de la fécondité. Par ailleurs, les recensements en Belgique et les enquêtes Famille en France contiennent des questions rétrospectives sur le nombre d'enfants déjà nés. Comparant les résultats de l'état civil et ceux issus de ces questions rétrospectives, à la fois en France et en Belgique, à des échelles nationale et infranationale et pour des générations de femmes nées dans la première moitié $d u X X^{e}$ siècle, Sandra BRÉE, Thierry EGGERICKX, Jean-Paul SANDERSON et Rafael COSTA mettent en évidence une forte cohérence de l'ensemble de ces données, à quelques exceptions près. La mise en commun de ces différentes sources permet des analyses de la fécondité des générations de femmes sur une longue période.
\end{abstract}

L'objectif de cet article est de vérifier la pertinence de l'exploitation des données individuelles et rétrospectives des recensements de la population en Belgique et des enquêtes Famille en France, pour étudier l'évolution de la fécondité des générations de femmes nées entre le début du $\mathrm{XX}^{\mathrm{e}}$ siècle et le début des années 1960. La genèse de cette recherche est née d'un constat : celui d'une relative méconnaissance, notamment en Belgique, des comportements de fécondité au cours de la première moitié du $\mathrm{XX}^{\mathrm{e}}$ siècle. Plusieurs raisons expliquent cette carence. D’une part, l'utilisation de données administratives

\footnotetext{
* Centre de recherche en démographie, Université catholique de Louvain, Belgique.

** Interface Demography, Vrije Universiteit Brussel, Pleinlaan 5, 1050 Brussels

Correspondance : Sandra Brée, Centre de recherche en démographie, Université catholique de Louvain, 1 place Montesquieu, B1348 Louvain-la-Neuve, Belgique, courriel : sandra.bree@uclouvain.be
} 
individuelles de moins de 100 ans n'est autorisée ni en Belgique ${ }^{(1)}$ ni en France. D'autre part, les données agrégées disponibles les années de recensement offrent des possibilités limitées pour une étude fine des comportements de fécondité.

La solution que nous proposons consiste à utiliser les données individuelles issues d'enquêtes et de recensements récents, et plus précisément les questions habituellement posées aux femmes sur leur vie féconde ${ }^{(2)}$. Cette approche, qui permet de reconstituer la vie génésique de différentes générations de femmes interrogées au moment de l'enquête ou du recensement, offre un potentiel analytique a priori prometteur. Outre la dimension diachronique, qui consiste à étudier les variations de la fécondité au fil du temps, elle permet de calculer des indicateurs fins et diversifiés - descendance finale, intervalles intergénésiques, âges moyens aux différentes naissances, parités atteintes, taux de fécondité par âge, etc. - qui ne s'obtiennent habituellement, pour les générations anciennes, que grâce aux méthodes de reconstitution des familles à des échelles très réduites (villages ou petites villes). Les paramètres de fécondité peuvent être croisés avec d'autres variables extraites des recensements et des enquêtes, telles que la situation matrimoniale, le niveau d'instruction ou encore le lieu de naissance. Un autre apport majeur de cette approche est de pouvoir prendre en considération la dimension spatiale de la fécondité à l'échelle des régions françaises, des arrondissements et même des communes en Belgique.

Mais l'exploitation de données rétrospectives pose un certain nombre de problèmes liés à la mémoire des répondants ou à l'importance des non-réponses, auxquels s'ajoutent les éventuels effets de sélection, puisqu'on ne peut pas prendre en considération les comportements des femmes sorties d'observation par décès et par émigration dans le cas de données rétrospectives. Les résultats des enquêtes dépendent également de l'échantillonnage et des éventuelles variables de pondérations proposées par les responsables des enquêtes. L'objectif est d'évaluer l'importance de ces biais et de valider ou non l'utilisation de ces données rétrospectives pour des études longitudinales de la fécondité. Par rapport aux quelques rares travaux qui se sont livrés à ce type d'exercice (Andersson et Sobolev, 2013; Neels, 2006; Van Bavel, 2014), les apports de notre recherche sont triples : en premier lieu de vérifier la cohérence des données pour des générations anciennes nées au début du $\mathrm{XX}^{\mathrm{e}}$ siècle et examiner comment les éventuels biais ont évolué au fil des générations; en deuxième lieu de prendre en compte deux pays, la Belgique et la France, disposant de deux sources d'observation différentes - respectivement des recensements et

(1) En Belgique, les registres de population de moins de 100 ans ne peuvent être consultés et exploités, sauf avec l'accord de la Commission de protection de la vie privée. Comme pour le XIX ${ }^{e}$ siècle, ces registres informatisés mais non centralisés ne permettent en pratique que des études sur de petites communes ou au mieux de petites villes, compte tenu de l'ampleur des tâches de dépouillement et d'encodage des informations.

(2) Il s'agit des questions sur le nombre total d'enfants qu'elles ont mis au monde et sur leurs dates de naissance respectives. 
des enquêtes - posant a priori des problèmes différents; et en dernier lieu de vérifier l'ampleur des biais lorsque les indicateurs sont calculés à l'échelle des régions en France et à celles des arrondissements et des communes en Belgique.

Après une première partie consacrée à une revue de la littérature, nous présentons en deuxième partie des tests de validation des données à l'échelle des deux pays. La troisième partie porte sur la validation de ces données rétrospectives pour réaliser des analyses dans des découpages administratifs spatiaux plus fins.

\section{I. État de la recherche}

Dans le cas de la Belgique, les études utilisant les données individuelles des registres administratifs sont inexistantes pour le $\mathrm{XX}^{\mathrm{e}}$ siècle, compte tenu notamment de la durée de prescription de 100 ans des données nominatives. À l'instar de l'ouvrage de Lesthaeghe (1977), la plupart des travaux sur la fécondité au $\mathrm{XX}^{\mathrm{e}}$ siècle reposent sur des données transversales et concernent des périodes encadrant les années des recensements (Damas et al., 1988; Masuy-Stroobant, 1976, 1977). D'autres se basent sur les enquêtes de fécondité (enquêtes à passages répétés) réalisées en 1966, 1971, 1976, 1983 et 1991 ${ }^{(3)}$. Si ces enquêtes permettent une compréhension fine des comportements de fécondité, les résultats ont peu de profondeur temporelle et une représentativité spatiale limitée aux trois régions du pays ${ }^{(4)}$.

Les données individuelles et informatisées issues des recensements de la population plus récents offrent des perspectives nouvelles pour l'étude de la fécondité au $\mathrm{XX}^{\mathrm{e}}$ siècle. En effet, à partir des questions posées aux femmes sur le nombre d'enfants nés vivants et leur(s) date(s) de naissance, il est possible de reconstituer la fécondité de plusieurs générations de femmes. Les travaux de Neels (2006, 2010), de Van Bavel (2014) et dans une moindre mesure la monographie du recensement de 1991 consacrée à la fécondité (Schoenmaeckers et al., 2002) sont les seuls à avoir exploité ces données de manière longitudinale.

Pour la France, il existe de nombreuses analyses conjoncturelles de la fécondité, en particulier pour la seconde moitié du $\mathrm{XX}^{\mathrm{e}}$ siècle (voir notamment les analyses de l'Insee et de l'Ined). Une étude très complète, intitulée " Un siècle de fécondité en France » (Daguet, 2002), retrace l'évolution de la fécondité au $X x^{e}$ siècle avec des indicateurs transversaux et longitudinaux, mais uniquement à l'échelle du pays et en utilisant principalement les données du recensement et des statistiques d'état civil. Par ailleurs, les résultats détaillés des enquêtes Famille de 1982 (Desplanques, 1985), 1990 (Lavertu, 1997) et 1999

(3) De nombreux articles sur ces enquêtes (notamment celle de 1966) ont été publiés dans la revue Population et Famille. On se référera également au rapport de Lodewijckx (1999) ainsi qu'à l'article de synthèse de Oris (2009).

(4) Les enquêtes de 1983 et 1991 ne concernent que la Flandre et Bruxelles. 
(Barre et Vanderschelden, 2004) proposent des tableaux de données détaillés sur différentes thématiques dont la fécondité, et certaines données sont fournies par génération. Des recherches ont également été menées sur la fécondité des générations en utilisant ces données (Desplanques, 1993; Daguet, 2000 ; Toulemon, 1995, 2001). Les données à l'échelle infranationale sont moins nombreuses. L'Insee publie depuis 1954 les « Données de démographie régionale », dont les résultats conjoncturels sont issus du croisement des données des recensements et de l'état-civil (Daguet 2005; Le Bras, 1995; Todd et Le Bras, 2014). En revanche, il n'existe pas, à notre connaissance, d'analyse longitudinale de la fécondité française à l'échelle infranationale.

Dans le cas de la France, et contrairement à la Belgique, l'approche rétrospective et longitudinale de la fécondité ne peut être menée directement à partir des recensements pour les générations nées après la Première Guerre mondiale, car la question du nombre d'enfants n'est plus posée après le recensement de $1946^{(5)}$. À partir de 1954, cette question figure dans les enquêtes Famille associées au recensement ${ }^{(6)}$, qui ne concernent qu'un échantillon de la population, et dont le principe est de suivre l'évolution des nouvelles formes familiales à travers un questionnaire biographique (tableau 1). La première enquête Famille a été organisée conjointement au recensement de 1954, puis ont suivi celles de 1962 ${ }^{(7)}$, 1975, 1982, 1990, 1999 (Desplanques, 2005) et 2011. Les trois premières enquêtes n'ont concerné que les femmes non célibataires, contrairement aux suivantes, et ne seront donc pas traitées ici.

Tableau 1. Éléments descriptifs des enquêtes Famille en France

\begin{tabular}{|c|c|c|c|c|}
\hline \multirow{2}{*}{ Date de l'enquête } & \multirow{2}{*}{ Génération } & \multirow{2}{*}{ État matrimonial } & \multicolumn{2}{|c|}{ Effectif } \\
\hline & & & Femmes & Hommes \\
\hline 1954 & 1899-1908 & Non célibataire & 52474 & Non enquêtés \\
\hline 1962 & \multicolumn{4}{|c|}{ Non diffusée } \\
\hline 1975 & 1910-1960 & Non célibataire & 247000 & Non enquêtés \\
\hline 1982 & 1917-1963 & Toute situation ${ }^{(a)}$ & 310000 & Non enquêtés \\
\hline 1990 & 1921-1971 & Toute situation ${ }^{(a)}$ & 340000 & Non enquêtés \\
\hline 1999 & -1981 & Toute situation ${ }^{(a)}$ & 278000 & 167000 \\
\hline 2011 & -1993 & Toute situation ${ }^{(a)}$ & 238000 & 121000 \\
\hline
\end{tabular}

(5) La question du nombre d'enfants vivants est posée dans les recensements à partir de 1886 (pour les seuls enfants légitimes en 1891). Les tableaux récapitulatifs fournissent le nombre d'enfants selon la durée du mariage ou l'âge du chef de famille, mais pas selon l'année de naissance des mères. En revanche, dans la statistique des Familles des recensements de 1931 et 1946, les informations sur le nombre d'enfants par femme selon l'année de naissance de la mère sont disponibles.

(6) Les individus sélectionnés reçoivent, en même temps que le questionnaire de recensement, un questionnaire spécifique qu'ils sont invités à remplir eux-mêmes et à remettre à l'agent recenseur.

(7) L'enquête de 1962 n'est pas considérée comme diffusable par l'Insee pour des raisons de qualité : le fichier ayant connu des problèmes d'archivage, il manque environ un tiers des enregistrements. 
Dans les deux premières enquêtes exploitables (1982 et 1990), seules les femmes de 18 à 64 ans ont été interrogées, ce qui donne des informations pour les générations 1917 à 1938 et 1921 à 1946 (générations des femmes âgées de 45 ans et plus). Dans les enquêtes de 1999 et de 2011, les femmes et les hommes de plus de 18 ans ont été interrogées, ce qui permet de reconstituer la vie génésique de générations plus anciennes (la femme la plus âgée est née en 1893 pour l'enquête de 1999 et en 1907 pour celle de 2011). Les données utilisées sont les données corrigées et post-stratifiées par les chercheurs en charge des différentes enquêtes, notamment parce que la population recensée dans les secteurs d'agents recenseurs peut différer de la population générale, en particulier à l'échelle d'une région où l'échantillon est concentré dans certaines zones géographiques (Barre et Vanderschelden, 2004). Pour les enquêtes de 1999 et de 2011, les pondérations préconisées ont été appliquées ${ }^{(8)}$.

Le principe de sondage des enquêtes de 1982 et 1990 est de tirer au sort des zones géographiques afin d'obtenir un échantillon représentatif au niveau régional couvrant $1 / 50^{\mathrm{e}}$ de la population, puis d'interroger toutes les femmes de ces zones. Pour les enquêtes de 1999 et de 2011, un échantillon représentatif de districts ${ }^{(9)}$ a été tiré au sort dans chaque région après un classement en fonction des caractéristiques sociodémographiques des quartiers ${ }^{(10)}$.

L'utilisation de données rétrospectives pour l'analyse de la fécondité peut être affectée par certains problèmes :

- Des erreurs de mémoire, tant pour le nombre d'événements déclarés que leur calendrier (date, âge). Elles concerneraient davantage les femmes les plus âgées au moment du recensement ou de l'enquête (Neels, 2006; Van Bavel, 2014). Il est très difficile de quantifier l'ampleur de ce biais, mais on peut supposer qu'il est très faible, compte tenu de l'importance d'une naissance dans la vie d'une mère (Anderson et Sobolev, 2013). Néanmoins, avec une mortalité infantile encore élevée avant $1940^{(11)}$, les femmes les plus âgées ont pu omettre la naissance d'un enfant décédé en très bas âge, entraînant ainsi une sous-estimation du nombre de naissances (Van Bavel, 2014).

- Des non-réponses qui excluent potentiellement des naissances de l'observation alors que les mères y sont incluses, entraînant ainsi un biais de correspondance entre le numérateur et le dénominateur et donc une sous-estimation potentielle de la fécondité (Neels, 2006). Pour éviter ce biais on peut exclure les femmes n'ayant pas répondu, mais on doit alors poser l'hypothèse qu'elles ont les mêmes comportements de fécondité

(8) Les pondérations appliquées sont celles recommandées dans la documentation technique à savoir poidsm5 pour les analyses à l'échelle nationale, poidms7 pour l'échelle régionale de 1999 et poids_ind pour 2011.

(9) Zones de collecte des agents recenseurs (définition Insee).

(10) Groupement de plusieurs îlots contigus à l'intérieur d'une même commune (définition Insee).

(11) En Belgique et en France, le quotient de mortalité infantile était d'environ 150 \%o en 1900 et de 65 à $75 \%$ en 1940 (Caselli et al., 1999). 
que les répondantes, et qu'il n'y a donc pas d'effet de sélection à ce niveau. Or, les taux de non-réponse augmentent avec l'âge (Insee, 1982, 1990, 2002, 2014; Neels, 2006; Van Bavel, 2014), sont plus élevés pour les populations de nationalité étrangère, dans les milieux les plus urbanisés (Neels, 2006), ainsi que chez les célibataires ou les personnes vivant seules ou sans enfant, les individus au chômage et les moins diplômés (Insee, 1982, 1990, 2002, 2014). Les non-réponses se différencient également selon la région de résidence (Insee, 1982, 1990, 2002, 2014).

- Des biais liés aux effets de sélection induits par la mortalité et l'émigration internationale (Andersson et Sobolev, 2013; Wunsch, 2001). Ils conduiraient plutôt à une surestimation de la fécondité calculée à partir de données rétrospectives. En effet, la plupart des études sur la fécondité des émigrants suggèrent que ceux-ci se caractérisent par une fécondité avant l'émigration un peu moins élevée que celle des non-émigrants (Anderson et Sobolev, 2013; Eggerickx et al., 2014). Par ailleurs, il a été démontré que les femmes célibataires et celles sans enfant se distinguaient par un risque de mortalité plus élevé par rapport aux femmes en couple et aux mères (Doblhammer, 2000). Enfin, dans le cadre d'une approche infranationale de la fécondité, les migrations internes et internationales peuvent également induire un biais de sélection car la vie génésique des femmes a pu ne pas se dérouler là où elles résidaient au moment de l'enquête ou du recensement. Mais la fécondité des migrantes se rapprocherait davantage de celles des femmes du milieu d'accueil que de celles des zones de départ (Eggerickx et al., 2014).

\section{L'utilisation de données rétrospectives de fécondité à l'échelle nationale}

En 1958, dans le rapport intitulé « Les tendances récentes de la fécondité dans les pays industrialisés ${ }^{(12)}$, les Nations unies soulignaient que «(..) en général, les limites observées dans les recensements examinés n'affectent pas réellement l'interprétation des données présentées, mais il ne faut pas les oublier ${ }^{(13)}$. Selon Neels $(2006,2010)$, qui utilise le recensement belge de 1991, les biais inhérents à l'utilisation de données rétrospectives sont très limités. Pour le démontrer, il présente une série de tests confrontant des indicateurs transversaux $^{(14)}$ et longitudinaux ${ }^{(15)}$ calculés d'une part à partir des données

(12) « Recent trends in fertility in industrialized countries».

(13) « (...) in most respects, the limitations observed in the censuses under consideration will not seriously affect the interpretation of the data presented, but they should be kept in mind (...)» (p. 59). Cette citation est incluse dans le chapitre V, « La fécondité des femmes mariées dans les données de recensement » («The fertility of married women according to census data»).

(14) Il s'agit des taux de fécondité par âge, de l'indice conjoncturel de fécondité, de l'âge moyen à la maternité ou encore de l'âge moyen à la maternité selon le rang de naissance.

(15) Il s'agit de la descendance finale, de l'âge moyen à la maternité et des probabilités d'agrandissement. 
du recensement et, d'autre part, à partir de celles de l'état-civil. Pour tous les indicateurs extraits de ces deux sources de données, l'ajustement des séries par année d'observation ou par cohorte est très bonne, voire " excellente " (p. 278). En résumé, « Les mesures de la fécondité calculées rétrospectivement à partir des données du recensement de 1991 sont fiables pour la période depuis 1960 , et pour les cohortes de femmes nées entre 1918 et $1951 »^{(16)}$ (Neels, 2006, p. 301).

Van Bavel (2014) a comparé les données du recensement belge de 1981 à celles de l'état civil, mais uniquement dans le cadre des descendances finales par génération. Pour les générations nées entre 1921 et 1944, les différences sont négligeables, généralement inférieures à 0,03 enfant. Pour les générations plus anciennes, nées entre 1901 et 1920, les indices calculés à partir du recensement sont systématiquement inférieurs à ceux estimés à partir de l'état civil, mais les écarts restent faibles, entre 0,05 et 0,08 enfant. Dans les recensements, ces différences peuvent être dues à une sous-déclaration plus importante des enfants chez les femmes plus âgées, davantage confrontées à des problèmes de mémoire, mais également à un taux de non-réponse à la question sur le nombre d'enfants nés vivants qui augmente avec l'âge. Dans l'état civil, au contraire, il peut y avoir une surestimation car y sont enregistrées toutes les naissances, y compris parfois des naissances de mères qui ne sont pas domiciliées dans la commune, la région ou le pays (Davie et Mazuy, 2010).

Les effets de sélection liés à l'émigration et à la mortalité dans les études rétrospectives ont été mesurés par Andersson et Sobolev (2013). La méthode utilisée consiste, dans le cas de la Suède et pour les années 1961-1999, à comparer deux bases de données, l'une prospective, l'autre rétrospective, extraites des registres de population. La première permet de mesurer la fécondité de l'ensemble de la population à partir de 1961, dont celle des femmes décédées ou émigrées lors de la période d'observation. La seconde se réfère uniquement aux femmes présentes en 1999 et exclut toutes les femmes sorties d'observation. Les résultats démontrent que l'omission des femmes décédées ou émigrées dans le cas d'une exploitation de données rétrospectives a des effets négligeables sur les mesures de la fécondité, même si celle-ci s’avère légèrement surestimée pour les générations les plus anciennes.

En résumé, ces trois études valident l'utilisation de données rétrospectives pour la mesure longitudinale de la fécondité à l'échelle nationale. Qu'en est-il des recensements belges et des enquêtes Famille françaises? Les mêmes biais sont-ils observés à léchelle nationale et infranationale? Et qu'en est-il lorsqu'on affine l'analyse en s'intéressant aux taux de fécondité par âge? Les analyses ciaprès consistent à vérifier la qualité des données utilisées en confrontant la

(16) « Measures of general fertility calculated retrospectively from the 1991 census data are fully robust in the period domain as far back as 1960 and for cohorts of women born between 1918 and 1951 ». 
fécondité des mêmes générations ${ }^{(17)}$ pour la Belgique (recensements de 1961, 1981 et 2001) et pour la France (enquêtes Famille de 1982, 1990, 1999 et 2011) ${ }^{(18)}$. La comparaison est également menée avec des séries longitudinales existantes construites à partir d'autres sources de données. Ces analyses permettront, si les écarts sont réduits, de valider l'utilisation de données rétrospectives.

\section{L'importance des non-réponses selon la génération de naissance}

L'un des premiers problèmes pouvant invalider l'exploitation des données rétrospectives extraites des recensements et des enquêtes Famille concerne l'importance des non-réponses. Celles-ci peuvent induire, d'une part, un biais de correspondance entre numérateur et dénominateur lorsque l'on inclut les mères n'ayant pas répondu à la question sur le nombre d'enfants nés vivants dans le calcul des indices, et d'autre part un biais de sélection, si l'on décide d'exclure ces mères alors qu'elles se distingueraient par des comportements particuliers de fécondité.

La figure 1 présente les taux de non-réponse à la question sur le nombre d'enfants nés vivants pour les recensements belges et les enquêtes Famille. Pour le recensement belge de 1981, le taux de non-réponse baisse progressivement de 4,7\% pour celle née en 1900 à moins de $1 \%$ pour la génération née en 1941 , confirmant les observations de Neels (2006) et Van Bavel (2013) selon lesquelles les réponses manquantes sont davantage le fait des femmes les plus âgées. Le recensement de 2001 présente un profil similaire, mais uniquement pour les générations nées jusqu'en 1947, alors que les générations plus récentes se caractérisent au contraire par une augmentation du taux de non-réponse, de 3,2 \% pour la génération 1948 à 5,2 \% pour celle née en 1961. Cette hausse de l'indice pourrait être liée aux tendances de l'immigration internationale qui, pour ces générations, privilégient le regroupement familial et donc une installation plus durable des familles immigrantes en Belgique. Or, l'étude de Neels (2006) a démontré que les taux de non-réponse à la question sur le nombre d'enfants nés vivants étaient sensiblement plus élevés chez les personnes de nationalité étrangère.

Par ailleurs, le recensement de 2001 présente des taux de non-réponse systématiquement supérieurs, pour toutes les générations, à ceux de 1981, révélant ainsi une moins bonne qualité des données. Celle-ci est probablement liée au mode de collecte pratiqué en 2001 où les questionnaires de recensement ont été transmis et retournés uniquement par voie postale, sans appui et contrôle des agents recenseurs (Deboosere et al., 2006), alors que c'était le cas en 1981. Il est donc préférable pour les générations nées avant 1940 d’utiliser les données du recensement de 1981.

(17) Les générations 1917-1922 pour la Belgique et 1926-1930 pour la France qui sont les premières générations pour lesquelles des données de différentes enquêtes ou recensements sont utilisables.

(18) Les données des recensements belges ont été obtenues auprès de la Direction générale de la statistique (DGS) et les données des enquêtes françaises auprès du réseau Quetelet. 
Figure 1. Évolution du taux de non-réponse à la question sur le nombre d'enfants nés vivants, par génération et date de recensement (Belgique) ou date d'enquête (France)

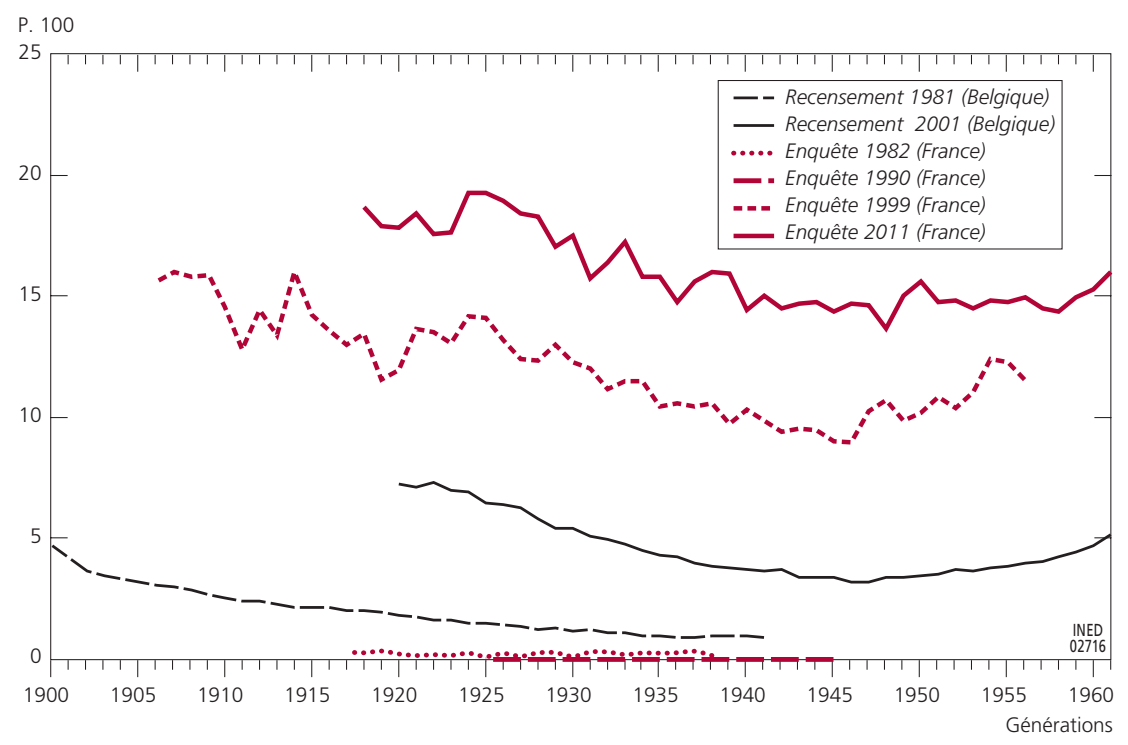

Sources : Recensements 1981 et 2011, Belgique; enquêtes Famille 1982, 1990, 1999, 2011, France.

Dans le cas des enquêtes Famille, le contraste est très marqué entre celles de 1982 et de 1990 qui présentent un taux de non-réponse insignifiant à la question du nombre d'enfant nés vivants(19), alors que l'indice fluctue entre $9 \%$ et $16 \%$ pour l'enquête de 1999, et entre $14 \%$ et $19 \%$ pour celle de 2011 . L'écart entre ces deux dernières enquêtes doit cependant prendre en compte le taux global de non-réponse, de 21 \% pour l'enquête de 1999 et de 17 \% pour celle de 2011. Ces taux sont comparables à ceux d'autres enquêtes non obligatoires, mais très supérieurs aux taux de non-réponse des enquêtes Famille de 1982 et 1990 d'environ 3 \% (Insee, 2002). L'enquête Famille était obligatoire jusqu'en 1990, mais en 1999, l'importance du volet rétrospectif de l'enquête a conduit ses concepteurs à ne pas demander l'obligation de réponse (Héran, 2005). Ce changement explique très probablement la différence sensible entre les taux de non-réponse des enquêtes de 1982 et 1990 et celles plus récentes de 1999 et 2011.

Au-delà de ces différences, l'évolution par génération des taux de nonréponse des enquêtes de 1999 et de 2001 est analogue à celle du recensement belge de 2001. Pour les générations nées avant 1946-1947, les taux de nonréponse augmentent avec l'âge, alors que le relèvement de l'indice pour les générations plus récentes pourrait, comme dans le cas belge, être attribué à un effet de l'immigration internationale (Neels, 2006).

(19) Il n'est pas précisé dans le fichier si la variable correspondante a été corrigée au préalable. 


\section{Les descendances finales et les taux de fécondité par âge d'après différentes sources}

Les figures 2 et 3 comparent les descendances finales (à 50 ans) par génération quinquennale, extraites des recensements de la population belge de 1961, 1981 et 2001 ${ }^{(20)}$ et des enquêtes françaises de 1982, 1990, 1999 et 2011. Deux séries d'indicateurs longitudinaux sont présentés : d'une part, les estimations proposées par Festy (1979) pour les générations de femmes nées entre 1816 et $1880^{(21)}$ pour la Belgique et entre 1831 et 1945 pour la France et, d'autre part, les valeurs annuelles fournies par Sardon (1990a, 1990b) pour les générations nées entre 1906 et 1954 pour la Belgique et entre 1901 et 1956 pour la France. Ces deux séries reposent sur une transposition longitudinale de taux de fécondité par âge (ou groupe d'âges) transversaux construits à partir de méthodes indirectes (Festy, 1979) et de données d'état civil (Sardon, 1990a, 1990b). Toulemon (2001) et Daguet (2002) ont, par ailleurs, déjà estimé la

Figure 2. Confrontation des descendances finales selon différentes sources de données: le cas de la Belgique

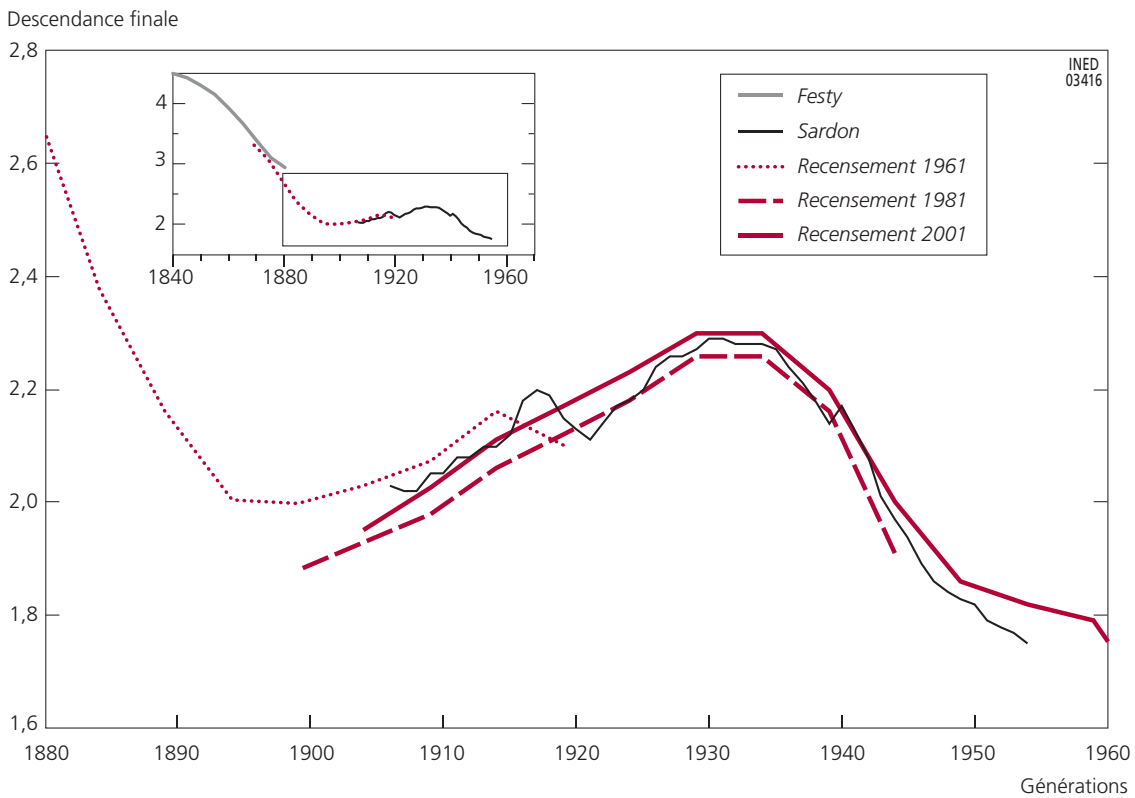

Sources : Festy 1979, Sardon 1990a et 1990b, recensements belges.

(20) En Belgique, le recensement de la population de 2001 a été dénommé « Enquête socio-économique $2001 »$ (ESE 2001).

(21) Il s'agit d'une lecture en diagonale des taux quinquennaux de fécondité générale calculés à partir des données d'état civil pour les périodes 1846-1850 à 1896-1900. Ces estimations sont basées sur une méthode indirecte expliquées dans l'ouvrage de Festy (1979) aux pages 209-211 pour la Belgique et page 335 pour la France. 
fécondité des générations françaises à partir du recensement de 1946 et des enquêtes Famille, après correction des non-réponses ${ }^{(22)}$.

Cette confrontation souligne la pertinence des indicateurs calculés sur la base des recensements. Pour la Belgique, les descendances finales du recensement de 1961 sont prolongées par la courbe des estimations de Festy, et les indices tirés des recensements de 1961, 1981 et 2001 se confondent avec ceux calculés par Sardon. Les différences sont très faibles et n'excèdent pas $3 \%$ entre les descendances finales calculées sur la base de l'état civil et celles construites à partir des recensements. Il en va de même pour la France (figure 3). Les trois premières enquêtes fournissent des résultats très proches de ceux de Sardon et de Festy, en particulier l'enquête de 1999, même pour les générations les plus anciennes (1900-1904). La reconstitution de la descendance finale par Toulemon et Daguet à partir du recensement de 1946 et des différentes enquêtes Famille offre les résultats les plus proches de ceux de Sardon et Festy. L'enquête de 2011, en revanche, sous-estime la fécondité. Il semble exister une sousdéclaration des enfants nés avant l'union en cours au moment de l'enquête, en particulier quand ces enfants ne vivent pas avec le couple (Mazuy et Toulemon,

Figure 3. Confrontation des descendances finales selon différentes sources de données : le cas de la France

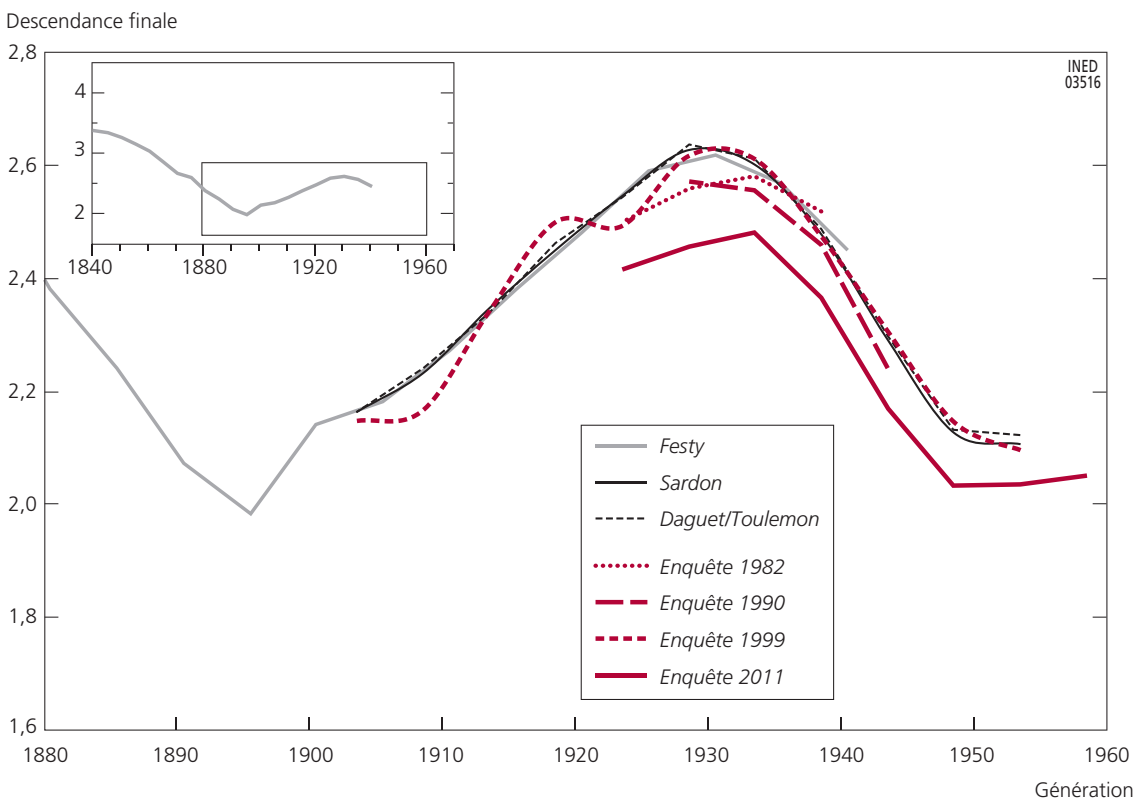

Sources: Festy, 1979; Sardon, 1990a, 1990b; Daguet, 2002; Toulemon, 2001 ; enquêtes Famille.

(22) Toulemon (2001) et Daguet (2002) ont estimé la descendance finale et les parités. Le but des recherches à venir est d'explorer d'autres indicateurs, et surtout d'utiliser ces sources pour des analyses individuelles, après les avoir validées au niveau agrégé, ce qui est l'objet de cet article. 
2013). Lanalyse des parités (distribution des femmes selon le nombre de leurs enfants) montre plus particulièrement une surestimation de l'infécondité et une sous-estimation des parités de 4 enfants et plus.

L'exercice peut être affiné en comparant, pour quelques générations, les taux de fécondité par groupes d'âges quinquennaux provenant des recensements et des enquêtes, aux séries proposées par Sardon (1990b) basées sur l'état civil. Pour la Belgique ${ }^{(23)}$, la concordance entre les courbes est quasi parfaite (figure 4) et confirme la très bonne correspondance, à l'échelle nationale, des indicateurs longitudinaux de fécondité extraits des données des recensements. Pour les générations les plus anciennes, nées entre 1922 et 1936, les taux de fécondité issus du recensement de 1981 s'ajustent mieux avec les indices proposés par Sardon (1990b). Mais les écarts avec les taux extraits du recensement de 2001

Figure 4. Confrontation des taux de fécondité par groupe d'âges quinquennaux selon différentes sources de données: le cas de la Belgique

Générations 1922-1926

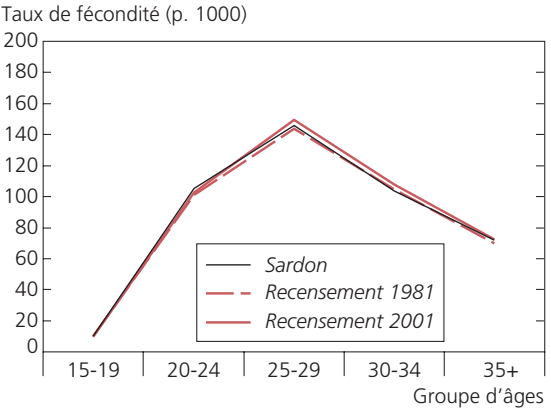

Générations 1932-1936

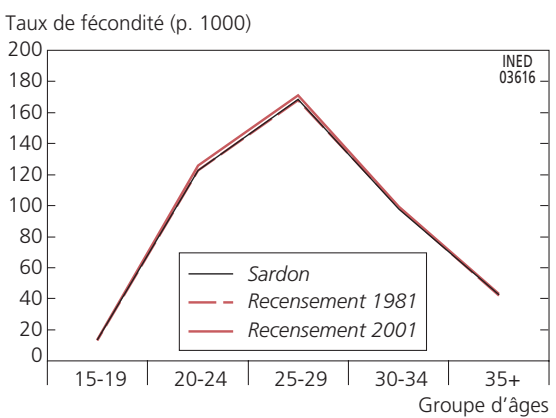

Sources : Sardon (1990b) et recensements.

Figure 5. Confrontation des taux de fécondité par groupe d'âges quinquennaux selon différentes sources de données : le cas de la France

Générations 1926-1930

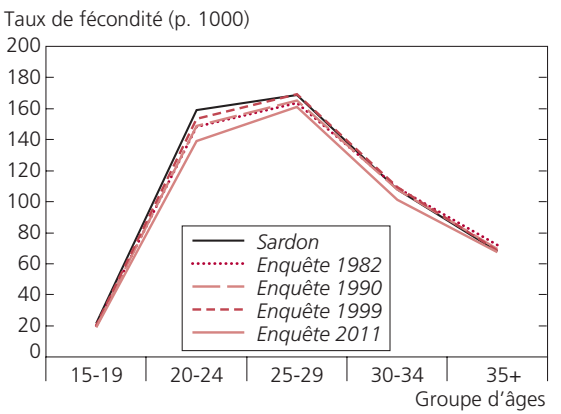

Générations 1936-1940

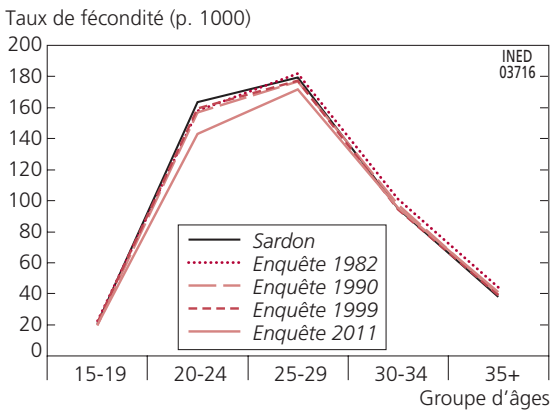

Sources : Sardon (1990b) et enquêtes Famille.

(23) Les résultats de Daguet sont très proches de ceux de Sardon pour la France et ne sont pas représentés ici. 
sont très faibles, n'excédant pas $4 \%$ pour le groupe d'âges des 30-34 ans de la génération née entre 1922 et 1926. Pour la France (figure 5), les taux s'ajustent moins parfaitement avec ceux de Sardon, en particulier pour les 20-24 ans (écarts de $6 \%$ en tenant compte de toutes les enquêtes mais de $3 \%$ si l'on exclut celle de 2011) et pour les générations les plus âgées au moment de l'enquête. Les résultats de l'enquête de 1999 sont les plus proches de ceux proposés par Festy et Sardon ${ }^{(24)}$, qu'il s'agisse des descendances finales ${ }^{(25)}$ et surtout des taux par âge ${ }^{(26)}$, et ce malgré l'âge des générations les plus anciennes. Les écarts entre les enquêtes sont également assez faibles, de $1 \%$ à $2 \%$ pour les $20-29$ ans si l'on exclut celle de 2011, et de $3 \%$ à $5 \%$ si on la prend en compte.

\section{Estimation des biais de sélection à partir des données rétrospectives}

L'exploitation des données rétrospectives extraites des recensements de la population ou des enquêtes pose d'emblée le problème des biais de sélection. On ne caractérise que les comportements de fécondité des femmes appartenant à une génération donnée et présentes au recensement. Or, qu'en est-il des comportements des femmes de cette même génération, mais sorties d'observation par décès ou par émigration? Comme il est très difficile de répondre à cette question, on pose généralement l'hypothèse d'indépendance entre les phénomènes selon laquelle ni la mortalité ni la migration n'influent sur la fécondité, ce qui implique que les décédées et les migrantes ont les mêmes comportements de fécondité que les femmes interrogées lors du recensement. Dans le cas contraire, les indices de fécondité calculés à partir des données du recensement ne fourniraient qu'une vue tronquée des comportements des générations. Andersson et Sobolev (2013) ont montré, dans le cas d'enquêtes rétrospectives à partir des registres de population suédois, que l'effet causé par l'omission de l'information relative aux individus qui ont migré ou sont décédés est négligeable, excepté dans le cas des immigrantes (légère surestimation de la fécondité réelle de rang 1 de cette population).

Pour la Belgique, on peut estimer l'ampleur ou l'impact de ces biais de sélection en comparant, pour une même génération de femmes, les descendances finales issues des recensements de 1981 et de 2001. Prenons l'exemple de la génération née entre 1917 et 1921 (tableau 2). En 1981, les 235000 femmes de cette génération étaient âgées de 60-64 ans. En 2001, cette génération ne compte

(24) Les écarts entre les différentes enquêtes s'élèvent à 2,5\% en moyenne pour la descendance finale (2,7\% avec Festy; $2,3 \%$ avec Sardon) et à 1,6\% pour les écarts entre les taux par âge tirés des enquêtes et les résultats de Sardon.

(25) Pour les descendances finales, les écarts avec Festy s'élèvent en moyenne à 3,7 \% pour les enquêtes de 1982 et 1990, 1 \% pour l'enquête de 1999 et $6 \%$ pour celle de 2011 ; et respectivement à $4,2 \%, 6,7 \%, 0,9 \%$ et $5,1 \%$ avec les données de Sardon.

(26) Pour les taux par âge, les écarts sont de moins de $4 \%$ entre les taux par âge issus de l'enquête de 1999 et ceux de Sardon pour la génération 1926-1930, et de moins de 8 \% pour la génération 19311935 (excepté pour le groupe d'âges 35 ans et plus). 
plus que 138000 femmes âgées de 80-84 ans. En d'autres termes, toute différence significative entre les indices de fécondité de cette génération calculés aux deux recensements révèlerait des biais imputables soit à la mémoire - celle-ci étant a priori moins fiable chez les plus âgées - soit à l'effet de sélection, puisque ne subsistent en 2001 que celles ayant échappé à la mort et n'ayant pas émigré ${ }^{(27)}$. Le tableau 2 montre que les écarts ne sont pas très importants et vont dans le sens d'une légère surestimation de la fécondité en 2001. A priori, un biais lié à la mémoire induirait plutôt une sous-estimation, or dans ce cas, on observe l'inverse. On pourrait dès lors conclure à un très faible effet de sélection lié à la migration et à la mortalité : les femmes ayant survécu et n'ayant pas migré seraient un peu plus fécondes que les autres.

Tableau 2. Effectifs de femmes et descendances finales

de mêmes générations, observées aux recensements belges de 1981 et 2001

\begin{tabular}{|c|c|c|c|c|c|c|c|}
\hline \multirow{2}{*}{ Génération } & \multicolumn{2}{|c|}{ Âge en } & \multicolumn{2}{|c|}{ Effectif de femmes } & \multirow{2}{*}{$\begin{array}{l}\text { Différence } \\
\text { (2001-1981) }\end{array}$} & \multirow{2}{*}{$\begin{array}{l}\text { Descendance } \\
\text { finale } 1981\end{array}$} & \multirow{2}{*}{$\begin{array}{l}\text { Descendance } \\
\text { finale } 2001\end{array}$} \\
\hline & 1981 & 2001 & 1981 & 2001 & & & \\
\hline $1917-1921$ & $60-64$ & $80-84$ & 234793 & 137527 & -97266 & 2,12 & 2,17 \\
\hline $1922-1926$ & $55-59$ & $75-79$ & 308689 & 226985 & -81704 & 2,18 & 2,23 \\
\hline $1927-1931$ & $50-54$ & $70-74$ & 312154 & 260062 & -52092 & 2,26 & 2,30 \\
\hline 1932-1936 & $45-49$ & $65-69$ & 296648 & 264001 & -32647 & 2,26 & 2,30 \\
\hline 1937-1941 & $40-44$ & $60-64$ & 277179 & 255717 & -21462 & 2,16 & 2,20 \\
\hline
\end{tabular}

Si on poursuit cette analyse en examinant les taux de fécondité par âge, le résultat est similaire. Les courbes s'ajustent presque parfaitement (figure 6) et les écarts relatifs entre les taux de fécondité déduits des recensements de 2001 et de 1981 sont très faibles. Ils atteignent leur maximum (6\%) pour la génération des femmes nées entre 1917 et 1921 (figure 7). Les différences relatives s'atténuent progressivement entre les générations suivantes.

Même si les biais de sélection et de mémoire semblent négligeables et n'invalident donc pas l'utilisation de ces données rétrospectives, les descendances finales et les taux de fécondité par âge calculés sur la base du recensement de 2001 sont systématiquement un peu plus élevés que ceux extraits du recensement de 1981. En d'autres termes, les femmes sorties d'observation par mortalité ou émigration se distingueraient par une fécondité très légèrement inférieure aux autres, un résultat similaire à celui obtenu par Andersson et Sobolev (2013) sur la Suède.

(27) Nous ne savons rien des personnes de ces générations décédées ou émigrées avant 1981. Des différences entre les données des recensements de 1981 et de 2001 pourraient aussi être liées à l'immigration au cours de cette période, qui ne peut toutefois être que minime pour les générations nées avant la Seconde Guerre mondiale. Ainsi, la génération 1937-1941 est composée de femmes de 60-64 ans au recensement de 2001, et ayant potentiellement immigré après 40 ans, soit à des âges où l'immigration internationale est très faible (Eggerickx, 2006). 
Figure 6. Taux de fécondité par âge et par génération selon les recensements belges de 1981 et de 2001

Générations 1917-1921

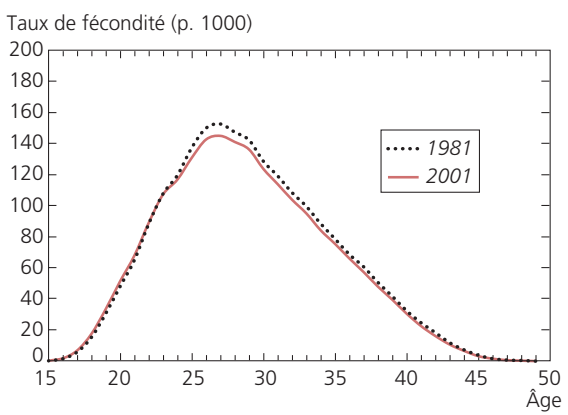

Générations 1932-1936

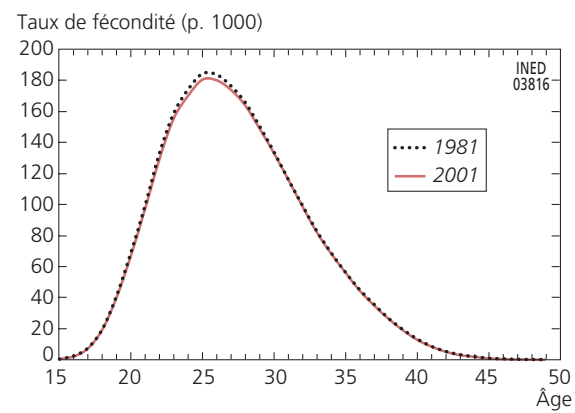

Sources : Recensements, Belgique.

Figure 7. Écarts relatifs entre les taux de fécondité par âge des recensements belges de 1981 et de 2001 et selon la génération des mères

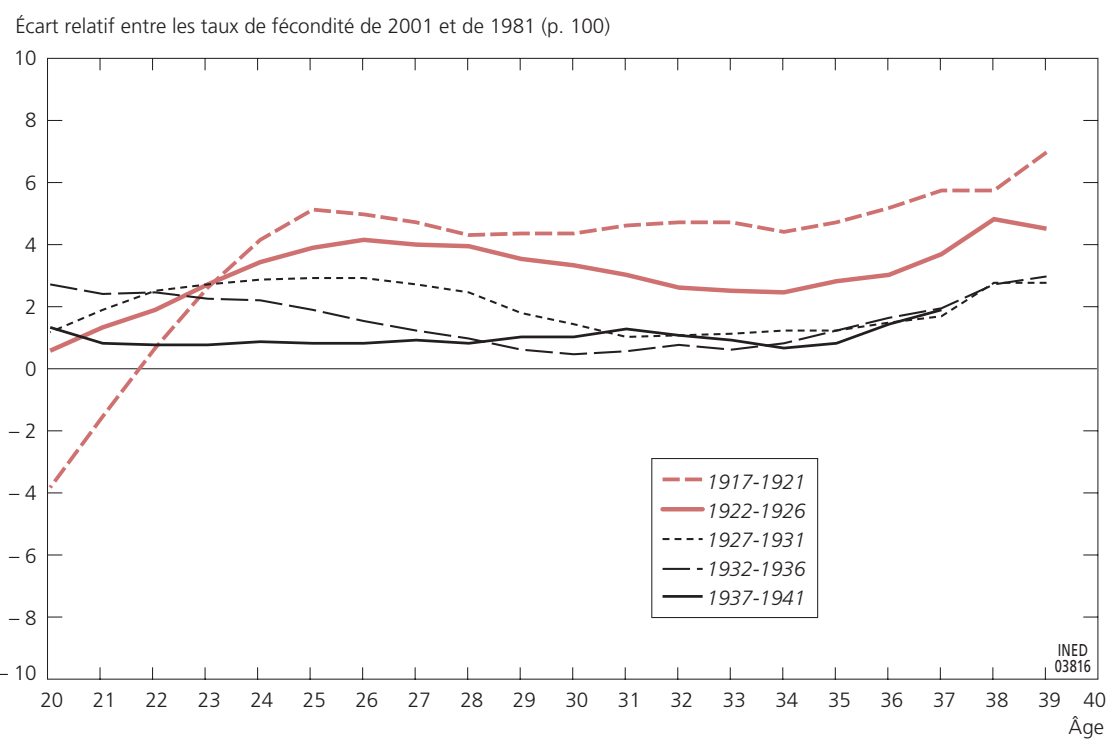

Note : Écart relatif : (taux de fécondité 2001 - taux de fécondité 1981)/ taux de fécondité 1981.

Sources : Recensements 1981 et 2001, Belgique.

Pour la France, la question se pose différemment, les enquêtes n'étant pas exhaustives. Les descendances finales sont très proches, quelle que soit l'enquête (écart moyen de $5 \%$, tableau 3) et il en va de même pour les taux par âge (figure 8), bien que les écarts soient plus importants que pour les recensements belges. Observons les générations 1926-1930 et 1931-1935 (figure 8) qui sont les seules pour lesquelles les quatre enquêtes sont disponibles. Globalement, 
Figure 8. Taux de fécondité par âge et par génération, enquêtes Famille de 1982, 1990, 1999 et de 2011, France

Générations 1926-1930

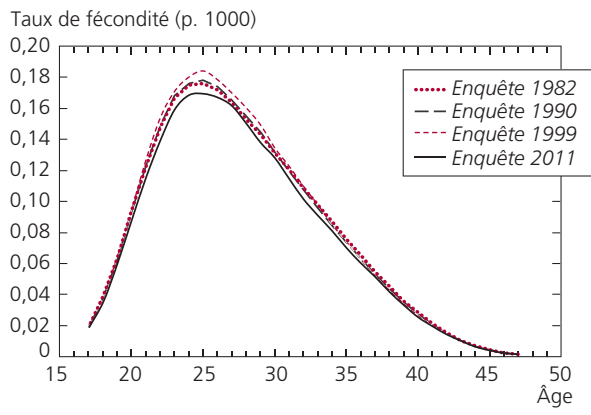

Générations 1931-1935

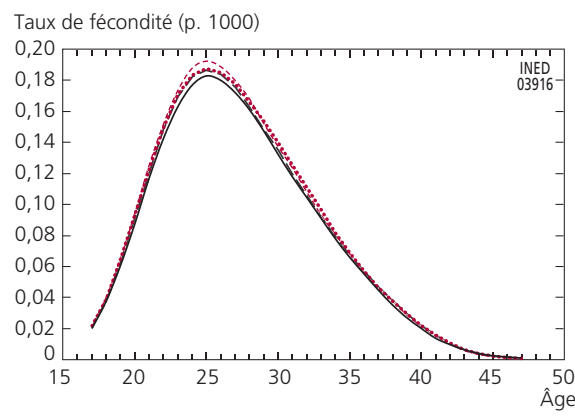

Note : Moyennes mobiles quinquennales sur les âges Sources: Enquêtes Famille, France.

l'enquête de 1990 sous-estime légèrement la fécondité par rapport à celle de 1982, et celle de 1999 surestime légèrement la fécondité par rapport à celle de 1990 (tableau 3). Quant à l'enquête de 2011, elle sous-estime la fécondité par rapport à toutes les autres, et assez fortement. Pour la génération 1926 1930, par exemple, les écarts entre les taux calculés à partir des différentes enquêtes par rapport à celle de 1982 sont, pour la plupart, inférieurs à $10 \%$ et même à 6 \% si l'on exclut l'enquête de 2011 (figure 9). On n'observe pas d'augmentation des écarts pour les générations les plus anciennes (comme pour la Belgique), c'est même l'inverse qui se produit parfois. En revanche, les écarts les plus importants se retrouvent pour les femmes âgées de plus de 35 ans.

Tableau 3. Effectifs de femmes et descendances finales de mêmes générations, observées aux enquêtes Famille de 1982, 1990, 1999 et 2011, France

\begin{tabular}{|c|c|c|c|c|c|c|c|c|c|}
\hline \multirow{2}{*}{ Générations } & \multirow{2}{*}{$\begin{array}{c}\text { Âge en } \\
1990\end{array}$} & \multicolumn{4}{|c|}{ Effectifs de femmes } & \multicolumn{4}{|c|}{ Descendance finale } \\
\hline & & 1982 & 1990 & 1999 & 2011 & 1982 & 1990 & 1999 & 2011 \\
\hline 1921-1925 & $65-69$ & 30067 & & 12950 & 5782 & 2,50 & & 2,49 & 2,39 \\
\hline 1926-1930 & $60-64$ & 30435 & 30663 & 14583 & 9660 & 2,56 & 2,57 & 2,62 & 2,46 \\
\hline 1931-1935 & $55-59$ & 30028 & 30552 & 15215 & 11907 & 2,58 & 2,56 & 2,61 & 2,48 \\
\hline $1936-1940^{(a)}$ & $50-54$ & 16862 & 28502 & 14492 & 12279 & 2,52 & 2,46 & 2,47 & 2,36 \\
\hline 1941-1945 & $45-49$ & & 28358 & 14668 & 12946 & & 2,24 & 2,31 & 2,17 \\
\hline 1946-1950 & $40-44$ & & & & 19935 & & & 2,14 & 2,03 \\
\hline 1951-1955 & $35-39$ & & & & 19725 & & & 2,09 & 2,03 \\
\hline
\end{tabular}


Figure 9. Écarts relatifs entre les taux de fécondité par âge des enquêtes Famille de 1982, 1990, 1999 et 2011 pour la génération 1926-1930, France

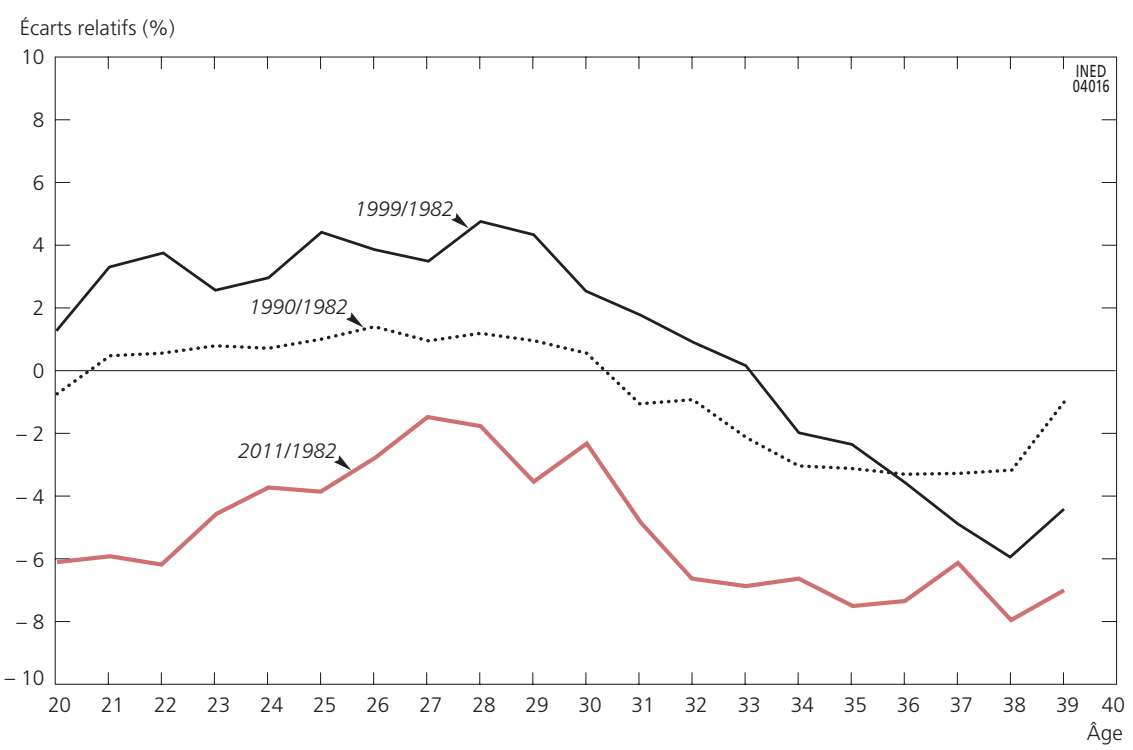

Exemple de calcul : Écart relatif 2011/1982 = (tx 2011 - tx 1982)/tx 1982.

Sources : Enquêtes Famille, France.

Le fait de travailler sur des enquêtes et non à partir des recensements exhaustifs peut, en soi, expliquer ces variations plus importantes que pour les recensements. Mais d'autre biais sont possibles. Les erreurs de mémoire peuvent difficilement être mises en cause puisqu'elles impliqueraient, par exemple, que les femmes, plus âgées en 1999 qu'en 1990 et 1982, déclarent davantage d'enfants (tableau 3), et à des âges légèrement supérieurs (figure 9) lorsqu'elles sont plus âgées. Ces différences de résultats s'expliquent probablement par l'échantillonnage qui diffère entre les deux premières ${ }^{(28)}$ et les deux dernières enquêtes ${ }^{(29)}$. L'effet de grappe est plus fort en 1999 et 2011 qu'en 1982 et 1990, et le taux de participation est légèrement inférieur (respectivement $79,4 \%$ et $83,4 \%$; Insee, 2002, 2014) alors qu'il était de $87 \%$ pour les éditions précédentes (Insee, 1982, 1990).

(28) Les enquêtes de 1982 et 1990 sont échantillonnées ainsi : « les zones où l'enquête a eu lieu sont des lots d'agent recenseur, c'est-à-dire des fractions de territoire comportant en moyenne 500 personnes confiées chacune à un agent recenseur. Ces zones ont été déterminées à la suite d'un tirage au sort conçu pour obtenir un échantillon qui soit représentatif au niveau régional » (Insee).

(29) Pour l'enquête de 1999, l'échantillonnage évolue : "Cet échantillon-il s'agissait là encore d'une première- devait en outre être conçu de sorte à être représentatif au niveau régional. À cet effet, on s'est basé sur des taux de sondage moyens de $1 / 170$ pour les hommes et $1 / 100$ pour les femmes, avec forcément un minimum de 5000 hommes et 8500 femmes s'agissant des régions pour lesquelles ces taux de sondage conduisaient à des effectifs inférieurs (sauf pour la Corse et le Limousin) » (Insee, 1982, 1990, 2002). 
En résumé, les tests réalisés valident l'utilisation des données rétrospectives des recensements de la population belge de 1961, 1981 et 2001 et des enquêtes Famille menées en France, pour une analyse longitudinale de la fécondité. Les erreurs de mémoire ne semblent pas affecter les résultats; quant aux biais de sélection liés à la mortalité ou aux migrations, ils ne semblent pas non plus avoir d'impact majeur sur les résultats. Des biais d'échantillonnage apparaissent cependant dans les enquêtes Famille liés à leur mode de recueil, par définition non exhaustif, et à leur mode d'échantillonnage.

Pour la France, nous recommandons de privilégier l'enquête Famille de 1999 pour une approche longitudinale et diachronique de la fécondité car ses résultats sont plus cohérents avec ceux calculés à partir d'autres sources de données. Pour la Belgique, compte tenu du plus grand nombre de femmes et d'un meilleur ajustement avec les séries d'indicateurs provenant de l'état civil, nous recommandons d'utiliser le recensement de 1981 pour couvrir les générations nées entre 1897 et 1936, et le recensement de 2001 pour les générations nées entre 1937 et 1966. Si nous y ajoutons les données plus partielles du recensement de 1961 -échantillon au $1 / 10^{\mathrm{e}}$ de la population et question unique sur le nombre d'enfants nés vivants-, il est possible de remonter jusqu'aux générations nées à partir de 1872, et donc de couvrir de manière longitudinale une large partie de l'histoire de la fécondité en Belgique lors des première et seconde transitions démographiques.

\section{L'utilisation de données rétrospectives de fécondité à l'échelle infranationale}

Les données rétrospectives issues du recensement et des enquêtes sont une source fiable pour reconstituer l'évolution de la fécondité au niveau national. Qu'en est-il à un niveau infranational? Comme précédemment, nous examinons d'abord l'importance des non-réponses avant de nous interroger sur l'impact des éventuels biais de sélection liés aux sorties d'observation par mortalité, migration internationale et migration interne.

\section{Les non-réponses}

La figure 10A présente les taux de non-réponse à la question sur le nombre d'enfants nés vivants au recensement belge de 1981 en distinguant les générations de femmes nées entre 1902 et 1916, et entre 1917 et 1931. Comme précédemment, et pour chaque arrondissement, le taux de non-réponse augmente avec l'âge des femmes interrogées. Pour les deux générations considérées, la grande majorité des arrondissements (80\%) ont un taux de nonréponse inférieur ou égal à la moyenne nationale $(2,7 \%$ pour la génération 1902-1916 et 1,6 \% pour la génération 1917-1931), soit des valeurs très modérées. Seule la situation de l'arrondissement urbain de Bruxelles dénote de 
Figure 10. Taux de non-réponse aux recensements belges de 1981 et 2001 à la question sur le nombre d'enfants nés vivants :

la situation des arrondissements

A. Selon les générations (recensement de 1981)

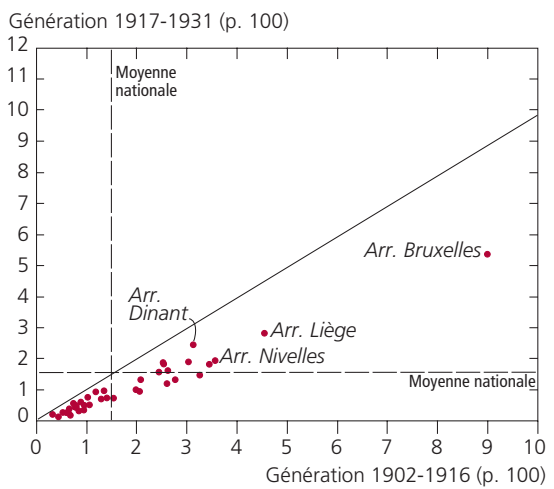

B. Selon les recensements de 1981 et de 2001 (générations 1917-1931)

Recensement 2001 (p. 100)

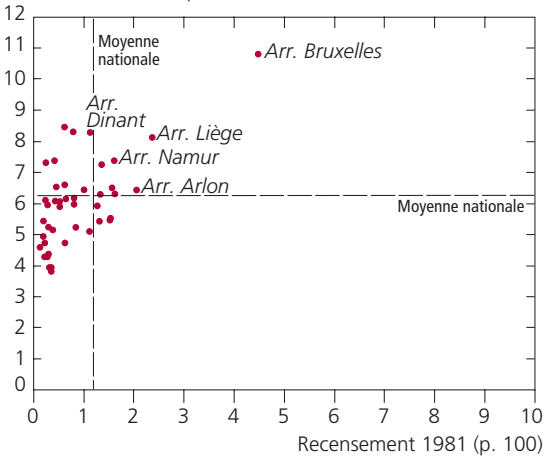

Note : Moyennes mobiles quinquennales sur les âges.

Sources : Recensements, Belgique.

l'ensemble avec des taux de non-réponse sensiblement supérieurs aux moyennes nationales.

La confrontation des recensements de 1981 et de 2001 pour la génération de femmes nées entre 1917 et 1931 (figure 10B) confirme, sans exception, la situation nationale, à savoir une augmentation du taux de non-réponse et donc, a priori, la moins bonne qualité du recensement 2001 pour les raisons évoquées précédemment (mode de collecte).

Les mêmes tendances caractérisent la situation des 589 communes. D’une manière générale, si l'on s'en tient au recensement de 1981, les taux de nonréponse sont tout à fait acceptables au niveau communal. Ainsi, pour la génération de femmes nées entre 1902 et 1916, 80 \% des communes (469 sur 589) ont un taux de non-réponse inférieur à la moyenne nationale et 7,5\% d'entreelles (45 sur 589), un taux supérieur à $5 \%$. Parmi celles-ci figurent la plupart des communes très urbanisées constituant l'agglomération de Bruxelles et la ville de Liège, où la forte présence de communautés étrangères peut expliquer, au moins partiellement, les taux de non-réponse plus élevés, conformément aux analyses réalisées par Neels (2010) dans le cas du recensement de 1991. Des taux de non-réponse supérieurs à la moyenne sont également observés dans de petites villes et des communes rurales, pour la plupart localisées en Wallonie.

En résumé, les non-réponses n'invalident pas, aux échelles sous-régionales et locales, l'utilisation des données rétrospectives des recensements belges pour l'étude de la fécondité. Néanmoins, il convient de garder à l'esprit que les taux de non-réponse ne se distribuent pas de façon aléatoire dans l'espace 
et que les milieux les plus urbanisés sont en moyenne davantage affectés par ce problème.

Dans les enquêtes Famille de 1999 et de 2011, le taux de non-réponse des femmes nées entre 1910 et 1939 à la question du nombre d'enfants nés vivants s'élève respectivement à $13 \%$ et $18 \%$, ce qui est comparable au taux de non-réponse d'autres enquêtes non obligatoires mais très supérieur aux taux de non-réponse des enquêtes Famille de 1982 et 1990 situés entre 0,15 \% et $0,23 \%{ }^{(30)}$. On retrouve les tendances observées au niveau national, à savoir que les générations les plus âgées au moment de l'enquête répondent moins à la question que les autres. Il faut noter que la part de non-répondantes augmente pour les générations nées après la Seconde Guerre mondiale.

Toutes les régions n'ont pas les mêmes taux de non-réponse. Dans l'enquête de 1999, les non-réponses sont toujours plus nombreuses en Corse, en Île-deFrance et en Provence-Alpes-Côte d'Azur, quelles que soient les générations; alors que dans l'enquête de 2011, seules deux régions se distinguent par leurs taux plus élevés : la Corse et l'Île-de-France (figure 11B). L'écart type du taux de non-réponse est plus élevé dans l'enquête de $2011(3,9)$ que dans celle de 1999 (2,5), ce qui signifie que les différences régionales sont plus accentuées dans l'enquête la plus récente.

Les enquêtes de 1999 et 2011 ont donc des taux élevés de non-réponse sur le nombre d'enfants nés vivants. La distribution de ces non-réponses n'est pas aléatoire dans les régions françaises (même si les écarts types entre régions sont assez réduits), et il faudra en tenir compte pour l'analyse de la fécondité française à l'échelle régionale à partir de ces enquêtes.

\section{Estimation des biais de sélection à partir des données rétrospectives à l'échelle régionale}

Pour les études rétrospectives de la fécondité au niveau régional, aux biais de sélection liés aux sorties d'observation par décès et émigration s'ajoutent ceux relatifs aux migrations internes. En effet, alors que l'appartenance territoriale des femmes est déterminée par leur lieu de résidence à la date des recensements et des enquêtes, leur vie génésique a pu se dérouler ailleurs, compte tenu des migrations effectuées avant le recensement ou l'enquête. Dans ce cas, il n'y aurait qu'une relation indirecte entre le lieu de résidence au recensement ou à l'enquête et la fécondité des femmes migrantes. Plus l'échelle géographique est fine, plus les migrations internes sont proportionnellement nombreuses et plus elles peuvent avoir un impact perturbateur important.

(30) Dans les fichiers des enquêtes de 1982 et de 1990, la variable originelle "nombre d'enfants * (libellée « ne »), qui existe pourtant dans le descriptif des variables, n'est pas fournie. Une variable appelée « variablo » décrite dans les fichiers de données comme « nombre d'enfants déclarés » semble la remplacer (les autres variables concernant le nombre d'enfants que les femmes ont eus consiste en un comptage des enfants reportés dans les tableaux concernant chaque enfant et ne comporte donc aucune non-réponse). 
Figure 11. Taux de non-réponse à la question sur le nombre d'enfants nés vivants dans les enquêtes Famille de 1999 et 2011

à l'échelle des régions françaises

A. Selon les générations (enquête de 1999)

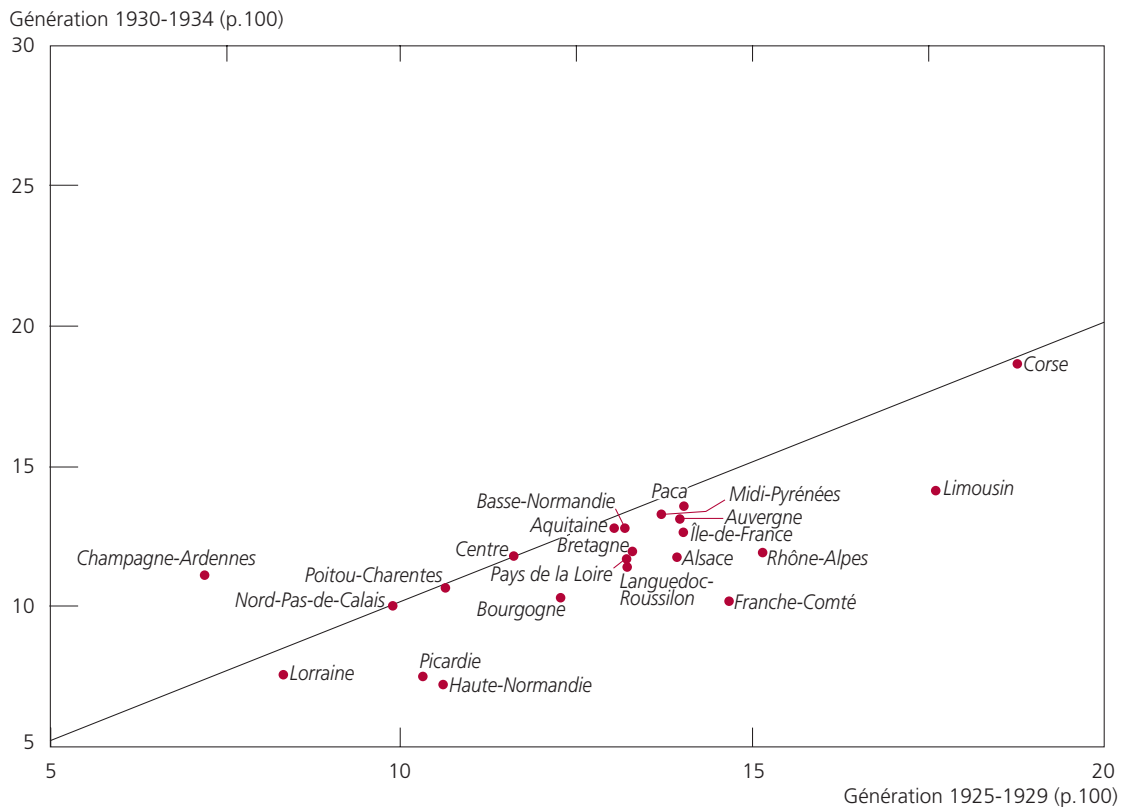

B. Selon les enquêtes de 1999 et de 2011 (générations 1925-1929)

Enquête 2011 (p.100)

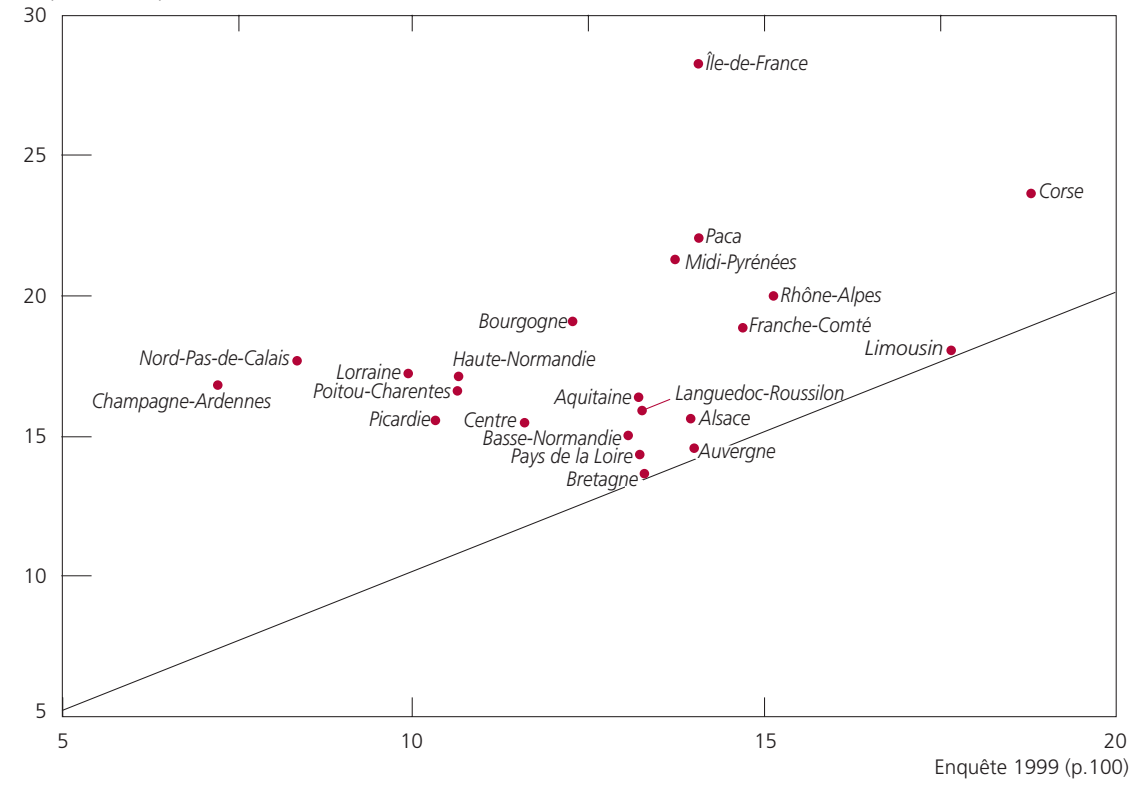

Sources: Enquêtes Famille 1982, 1990, 1999, 2011, France. 


\section{Le cas des régions françaises}

Avant de les évaluer, interrogeons-nous brièvement sur les perturbations que peuvent causer les migrations internes. Si celles-ci sont rares et se font sur de courtes distances, elles ne modifient pas les mesures de la fécondité. En revanche, dans le cas de nombreuses migrations de longue distance, la fécondité passée des femmes n'est pas forcément représentative de la fécondité de leur lieu de résidence au moment du recensement.

Pour la France, l'analyse des migrations est moins aisée qu'en Belgique, car il n'existe pas de registres de population comparables aux registres belges dans lesquels sont relevés les arrivées et les départs dans les communes (Desplanques, 1994). Différentes sources permettent néanmoins de connaître les déplacements de la population, et notamment l'analyse des migrations internes entre les recensements. Celles-ci ont augmenté de 1954 à 1975, puis diminué de 1975 à 1990, pour augmenter à nouveau à partir des années 1990 (Desplanques, 1994; Baccaïni, 2007).

Les analyses portant sur les migrations, à partir du recensement de 1975 jusqu'à celui de 2006, montrent que le sud de la France est un lieu d'attraction alors que le nord, et en particulier le nord-est, est une zone de départ (Baccaïni, 1993, 2001, 2009). Les mouvements migratoires diffèrent en fonction des groupes d'âges. Les jeunes adultes (20-30 ans) sont très mobiles car les causes de la mobilité résidentielle se cumulent : études, premier emploi, installation en couple et constitution de la famille. Ils sont attirés par des zones d'emploi, à savoir l'île-de-France - et plus récemment les régions alentour (Baccaïni, 2007) - et le sud du pays (Midi-Pyrénées, Paca, Rhône-Alpes, LanguedocRoussillon). Ces régions sont également très attractives pour les 30-39 ans. Quant aux migrations des individus de 60 ans et plus, le rejet du quart nord-est est évident, et en particulier de l'île-de-France et du Nord-Pas-de-Calais (Cribier et Kych, 1992; Baccaïni, 1993, 2001, 2009). Ces migrations, grandissantes à partir des années 1950 et de la généralisation des pensions, peuvent correspondre à un « retour chez soi », à une installation à la périphérie des grandes villes, moins chère que les centres-villes ou encore, pour les plus riches, à se retirer dans une maison de campagne, voire dans des villes d'eau ou de plaisance (Cribier et Kych, 1993). Cependant, ces migrations sont finalement assez peu nombreuses puisque seuls $4 \%$ des plus de 60 ans ont changé de département entre 2001 et 2006 (Baccaïni et Lévy, 2009). De plus, les distances parcourues sont très réduites : les individus nés entre 1911 et 1935 ont ainsi parcouru en moyenne $84 \mathrm{~km}$ lors de leurs migrations, et pour plus de la moitié, moins de 7,4 km (Baccaïni, 1994). L'analyse de la fécondité à l'échelle régionale ne peut donc être impactée par ces migrations inter-régionales car elles sont peu nombreuses. Elles ne permettent donc pas d'expliquer les écarts entre les différentes enquêtes Famille.

Les enquêtes Famille sont effectuées à partir de sondages représentatifs à l'échelle des 22 régions françaises, ce qui ne permet pas de considérer une 
échelle spatiale plus fine ${ }^{(31)}$. Néanmoins, le problème des petits effectifs se pose. Malgré le regroupement en générations quinquennales, les effectifs sont particulièrement réduits dans certaines régions, en particulier pour l'enquête de 1999. En effet, 310000 femmes sont interrogées en 1982 et 340000 en 1990, mais seulement 278000 en 1999 et 238000 en 2011. Pour ces deux dernières enquêtes, toutes les femmes de plus de 18 ans sont interrogées, ce qui contribue à faire diminuer le nombre de femmes par génération par rapport aux deux premières où seules les femmes de 18 à 64 ans l'étaient. En Corse par exemple, les effectifs par groupe d'âges ne sont jamais supérieurs à 130 femmes $^{(32)}$. Pour les enquêtes de 1982 et de 1990, les effectifs par région sont bien plus importants même si ceux de la Corse et du Limousin sont toujours assez faibles.

En comparant les descendances finales calculées à partir des différentes enquêtes Famille, il est possible d'évaluer la cohérence des trois séries de résultats par une analyse de corrélation. Ce croisement présente des résultats très homogènes pour les trois premières enquêtes (tableau 4), malgré le changement d'échantillonnage entre les enquêtes ${ }^{(33)}$. Les coefficients de corrélation $r$ sont particulièrement élevés entre les enquêtes de 1982 et $1990(r=0,94$ en moyenne) et celles de 1990 et 1999 ( $r=0,90$ en moyenne). Les coefficients entre l'enquête de 2011 et les 3 autres enquêtes sont globalement moins élevés (même pour les générations les plus récentes) mais ils sont presque toujours au-dessus de 0,80.

Tableau 4. Coefficient de corrélation des descendances finales régionales entre les enquêtes Famille de 1982, 1990, 1999 et 2011, France

\begin{tabular}{|l|c|c|c|c|c|c|}
\hline \multirow{2}{*}{ Générations } & \multicolumn{7}{|c|}{ Enquêtes Famille } \\
\cline { 2 - 7 } & $1982-1990$ & $1982-1999$ & $1982-2011$ & $1990-1999$ & $1990-2011$ & $1999-2011$ \\
\hline $1920-1924$ & & 0,79 & 0,91 & & & 0,63 \\
$1925-1929$ & 0,94 & 0,89 & 0,87 & 0,86 & 0,85 & 0,82 \\
$1930-1934$ & 0,96 & 0,88 & 0,87 & 0,92 & 0,89 & 0,86 \\
$1935-1939$ & 0,92 & 0,87 & 0,93 & 0,91 & 0,85 & 0,81 \\
$1940-1944$ & & & 0,90 & 0,91 & 0,83 \\
$1945-1949$ & & & & & 0,79 \\
$1950-1954$ & & & & & 0,88 \\
\hline Sources : Enquêtes Famille 1982, 1990, 1999, 2011, France. \\
\hline
\end{tabular}

(31) Les enquêtes de 1999 et 2011 fournissent également le département de résidence des individus interrogés, mais les échantillons ne sont pas censés être représentatifs à cette échelle.

(32) Huit autres régions (Haute-Normandie, Centre, Basse-Normandie, Bretagne, Champagne-Ardenne, Lorraine, Midi-Pyrénées et Limousin) comptent moins de 500 femmes par groupes quinquennaux d'âge pour les générations nées avant 1945.

(33) Voir la note 25. 
Concentrons-nous sur les générations 1925-1929, 1930-1934 (figure 12) et 1935-1939, les seules pour lesquelles les quatre enquêtes sont disponibles. À l'échelle des régions, comme à l'échelle nationale, les résultats des enquêtes de 1982 et de 1990 sont les plus proches (écarts relatifs moyens des descendances finales régionales inférieurs à $4 \%$ ), et les résultats des trois premières enquêtes sont assez éloignés de ceux de 2011 (toujours supérieurs à $5 \%$ ), en particulier celle de 1982. Aucun schéma spatial n’apparaît clairement et il est donc probable que les écarts soient liés à l'échantillonage des enquêtes qui change (voir note 31). Quant à l'enquête de 2011, elle sous-estime globalement la fécondité par rapport aux autres, mais sans qu'aucun schéma régional n’apparaisse vraiment.

Figure 12. Écarts relatifs entre les descendances finales des différentes enquêtes Famille pour la génération 1930-1934, France
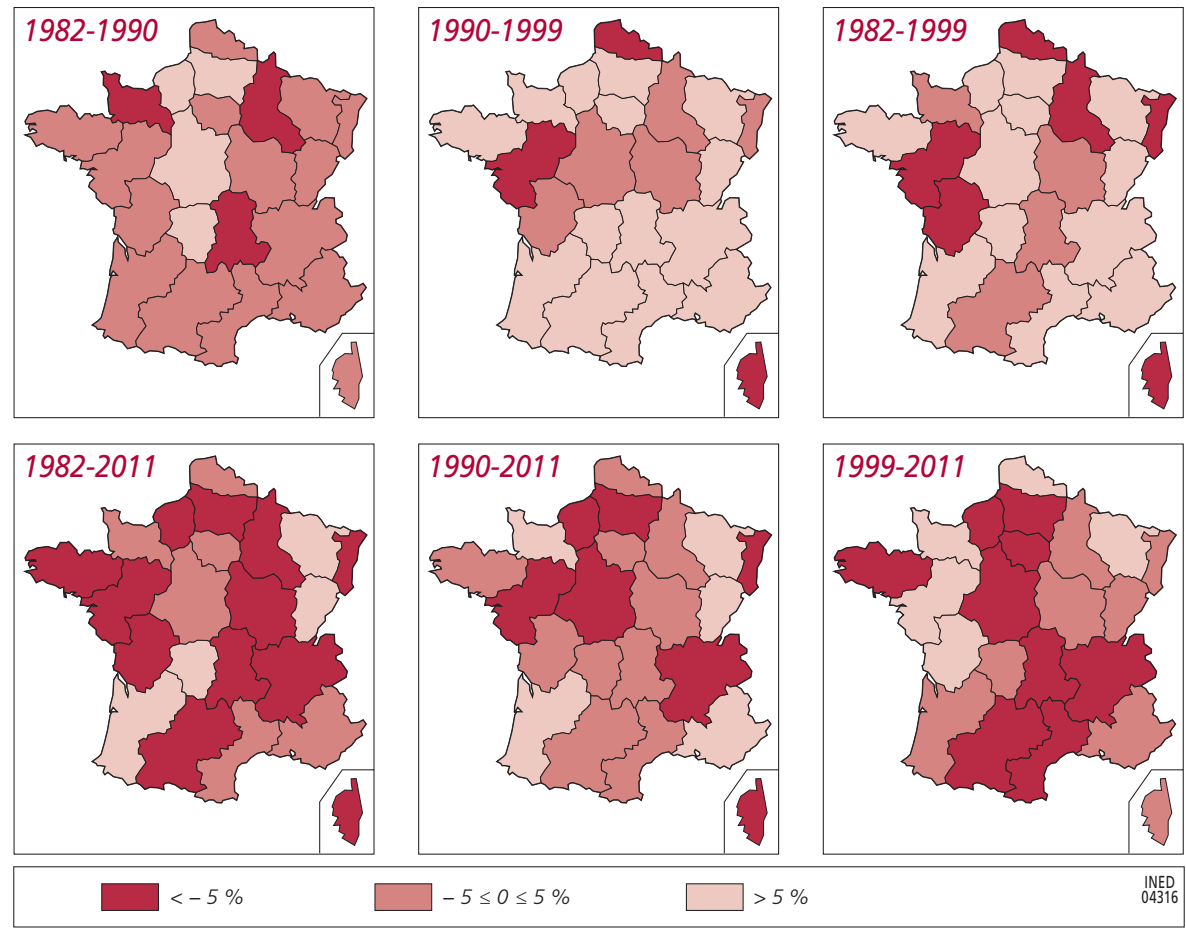

Note : L'écart 1982-1990 est calculé comme $\left(\mathrm{X}_{1990}-\mathrm{X}_{1982}\right) / \mathrm{X}_{1982}$. Sources : Enquêtes Famille, France.

Qu'en est-il des taux de fécondité par âge à l'échelle des régions? Les écarts relatifs entre les différentes régions dans les quatre enquêtes sont plus importants qu'au niveau national, ce qui s'explique notamment par la faiblesse des effectifs. À 25 ans, les écarts les plus élevés sont mesurés entre les enquêtes 1999 et 2011 (comme à l'échelle nationale) et les plus faibles entre 
les enquêtes 1990 et 2011 à l'échelle régionale (pour rappel, les écarts les plus faibles à l'échelle nationale sont ceux mesurés entre les enquêtes de 1982 et 1990). Les écarts relatifs moyens à 25 ans sont de l'ordre de $5 \%$ entre les enquêtes de 1990 et 2011 (écart type de 4,8) pour les générations 19201924, 1930-1934 et 1935-1939, et de 10 \% entre les enquêtes de 1999 et 2011 (écart type de 6,6).

Les taux par âge de l'enquête de 1999 sont souvent légèrement supérieurs à ceux des deux enquêtes précédentes (figure 13) alors que ceux de l'enquête de 2011 sont plutôt inférieurs. Mais comme l'exemple du Nord-Pas-de-Calais le montre, les tendances peuvent être inversées. Par ailleurs, la répartition spatiale des écarts entre les descendances finales calculées à partir des différentes enquêtes n'est pas la même que celle des écarts entre les taux à 25 ans. Pour les analyses ultérieures, il sera donc important de tenir compte de l'ensemble des enquêtes disponibles, en appréciant à leur juste mesure les écarts qui, à quelques exceptions près, sont assez réduits.

Figure 13. Taux de fécondité par âge de la génération 1930-1934 pour quelques régions de France selon les quatre enquêtes Famille

Île-de-France

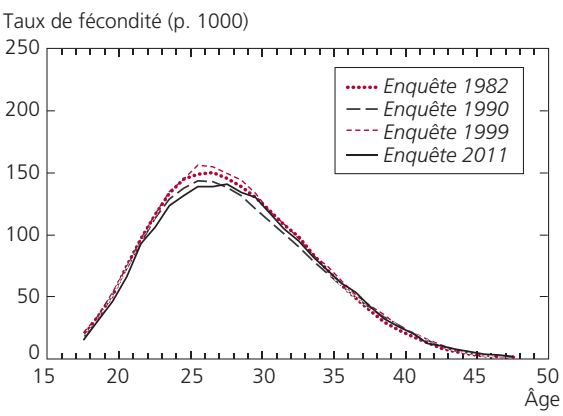

Haute-Normandie

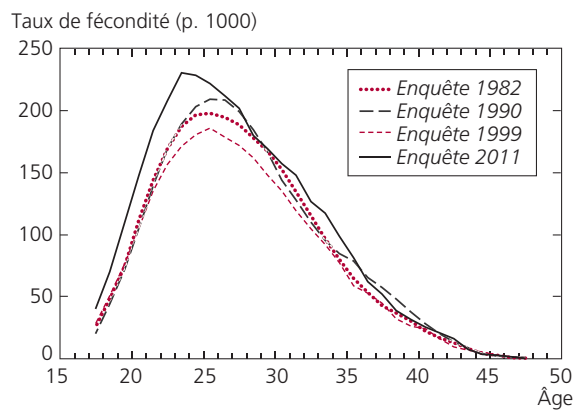

Languedoc-Roussillon

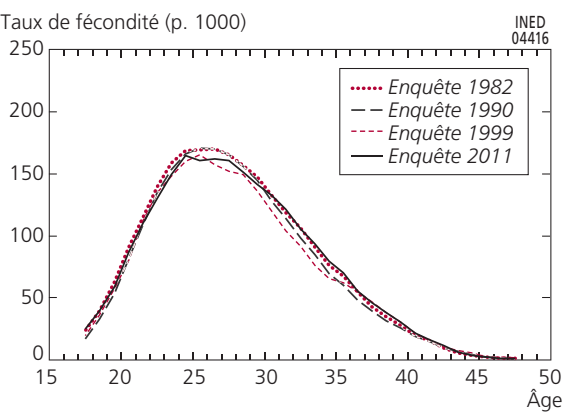

Nord-Pas-de-Calais

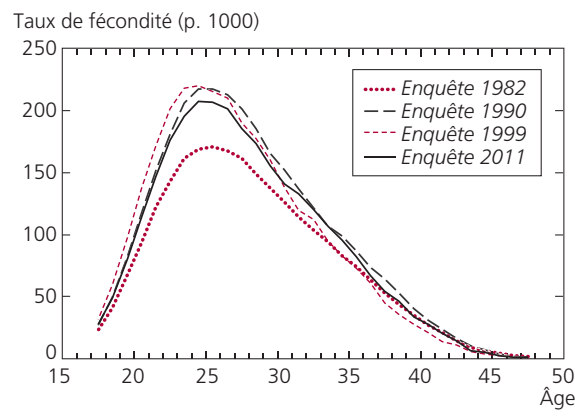

Note : La génération 1930-1934 est la seule pour laquelle les quatre enquêtes peuvent être utilisées. Les régions ont été sélectionnées de manière à montrer deux des meilleures corrélations entre les enquêtes (Île-de-France et Languedoc-Roussillon) et deux des moins bonnes (Haute-Normandie et Nord-Pas-de-Calais).

Sources : Enquêtes Famille, France. 


\section{Les arrondissements belges}

Pour la Belgique, plusieurs arguments peuvent être avancés en faveur de la pertinence d'une approche rétrospective et spatiale de la fécondité.

Premièrement, les spécificités des comportements migratoires en Belgique confortent l'utilisation de ces données. La plupart des migrations s'effectuent en effet, aujourd'hui comme hier, sur de petites distances à l'intérieur même des arrondissements. Ainsi, si on dénombre environ 1000000 de changements de résidence chaque année, la moitié de ceux-ci se déroulent à l'intérieur des 589 communes. Parmi les 500000 migrations intercommunales, seules 200000 impliquent un changement d'arrondissement (43 arrondissements) et 65000 un changement de région (11 régions) (Eggerickx et al., 2012). En outre, après l'âge de 25 ans, les taux de migration intercommunale ou interarrondissement diminuent très rapidement, car l'accès à la propriété et la constitution de la famille entraînent une plus grande stabilité résidentielle (Eggerickx et al., 2012).

Par ailleurs, une étude récente (Eggerickx et al., 2014) a montré que les migrations internes ont peu d'impact sur la fécondité. À partir du couplage des données des recensements de 1981 et de 2001, les auteurs ont analysé les générations de femmes nées entre 1952 et 1966 (celles dont une grande partie de la vie féconde s'est déroulée entre les deux recensements). L'étude est basée sur une répartition de l'espace communal belge en 6 types homogènes de communes selon l'évolution de leur fécondité ${ }^{(34)}$. Au sein de chaque type de commune, nous avons distingué deux groupes de femmes :

- les femmes n'ayant pas changé de type de commune durant leur vie féconde, c'est-à-dire celles qui permettent de calculer simplement la fécondité du type de commune de résidence en 2001 ;

- les femmes ayant migré pendant leur vie féconde, c'est-à-dire le groupe qui pourrait perturber le calcul de la fécondité dans le cadre d'une approche rétrospective et spatiale.

Il en ressort que le groupe perturbateur a une influence minime sur les indicateurs de fécondité dans tous les types de communes. L'inclusion de ce groupe dans les mesures rétrospectives de fécondité au lieu de résidence implique, pour la génération 1952-1956 par exemple, une surestimation systématique de la descendance finale en moyenne de l'ordre de 0,01 enfant par femme et une surestimation de l'âge moyen à la première maternité de 0,3 an. Ces analyses ont également montré l'existence d'un effet du territoire d'accueil sur la fécondité des migrantes : dans tous les types de communes, les comportements de fécondité des migrantes ressemblent plus aux comportements du lieu d'accueil qu'à ceux du lieu de départ. Cette étude, basée sur une typologie

(34) Il s'agit d'une typologie de communes élaborée à partir d'une méthode de classification hiérarchique (méthode de Ward) sur la base d'une série d'indicateurs transversaux de calendrier et d'intensité de la fécondité allant de 1971 à 2005. Cette typologie a été détaillée dans Costa et al. (2010). 
de communes et sur des générations assez récentes, démontre que les migrations internes ne semblent donc pas perturber l'observation de la fécondité de manière rétrospective. Qu'en est-il pour les générations plus anciennes, dans le cadre du découpage administratif classique par arrondissement?

Comme nous l'avons démontré dans la deuxième partie, à génération identique, la confrontation des données des recensements de 1981 et de 2001 à l'échelle nationale confirme l'hypothèse d'indépendance entre la fécondité d'une part, et la mortalité et les migrations internationales d'autre part. Au niveau des arrondissements, les migrations internes constituent un autre biais de sélection potentiel, d'autant que leur poids démographique est loin d'être négligeable, notamment pour les « espaces » les plus urbanisés (Eggerickx et al., 2012) et que ces migrations interarrondissements risquent d'être corrélées avec le comportement fécond, davantage que les migrations interrégionales en France.

Pour une même génération de femmes, toute différence significative entre les indices extraits des recensements de 1981 et de 2001 mettrait en évidence un biais de sélection important, traduisant des comportements différenciés entre les populations observées et les populations sorties d'observation. Comme lillustre la figure 14, les corrélations entre les séries provenant des deux recensements sont très élevées : pour toutes les générations pour lesquelles le croisement entre les données de 1981 et de 2001 est possible, le coefficient de corrélation est toujours proche de 0,99. Le même exercice a été réalisé entre les données de 1961 et de 1981, et là aussi, la valeur des coefficients de corrélation est très élevée, toujours supérieure à 0,95 . En d'autres termes, les descendances finales pour une même génération, calculées en 1981 et en 2001 sont quasiment identiques, et ce même pour les arrondissements de petite taille de la province du Luxembourg (au sud de la Belgique). À l'échelle des arrondissements, les biais de sélection

Figure 14. Comparaison des descendances finales par arrondissement aux recensements belges de 1981 et de 2001

Générations 1912-1916

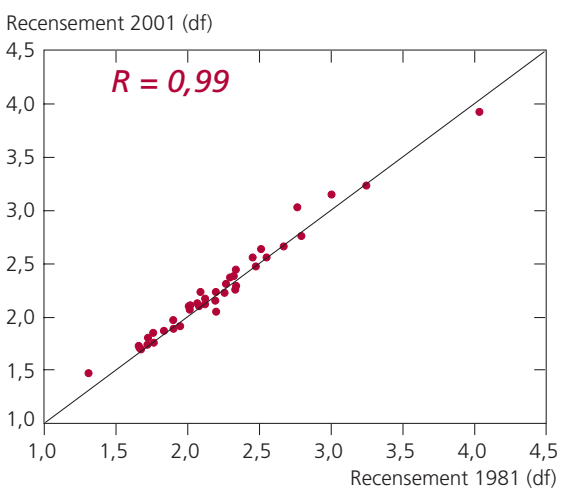

Générations 1932-1936

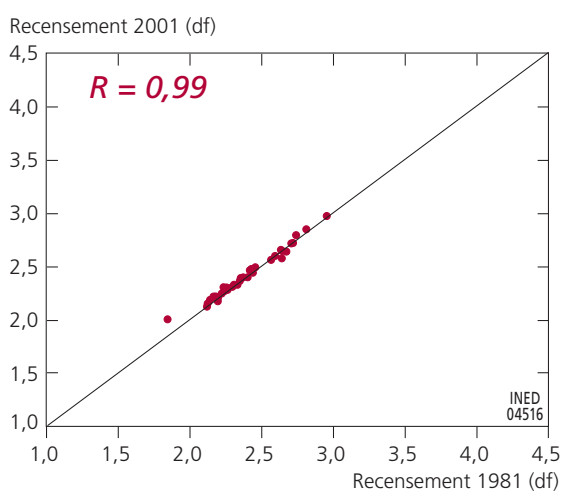

Sources: Recensements de 1981 et 2001, Belgique. 
éventuels n'invalident donc pas le recours aux données rétrospectives de fécondité provenant des recensements de la population.

C'est pour l'arrondissement urbain de Bruxelles que les écarts entre les recensements sont les plus marqués : pour la génération de femmes nées entre 1912 et 1916, la descendance finale calculée sur base du recensement de 1981 est de 1,31, alors que le recensement de 2001 donne un indice de 1,48. Pour la génération née entre 1932 et 1936, les valeurs de cet indicateur sont respectivement de 1,85 et de $2,00^{(35)}$.

Qu'en est-il des taux de fécondité par âge? La figure 15 compare les taux de fécondité des générations 1922-1926 et 1932-1936 pour l'arrondissement de Bruxelles, là où les différences de descendances finales entre 1981 et 2001 sont les plus importantes. On observe pour toutes les générations un croisement des courbes de taux de fécondité par âge indiquant un calendrier plus précoce en 2001. Les différences de taux sont faibles et pourraient s'expliquer par la présence en 2001 d'une population immigrée sensiblement plus importante qu'en 1981 à Bruxelles et se caractérisant par un calendrier de fécondité plus précoce (Eggerickx, 2006). Globalement, les écarts entre les courbes sont faibles et ont tendance à diminuer au fil des générations. Pour la génération 1922-1926, les écarts maximum sont observés entre 21 et 25 ans, mais n'excèdent pas $8 \%$ o en valeur absolue du taux de fécondité ou $10 \%$ en valeur relative. Pour la génération 1932-1936, c'est entre 31 et 35 ans que les différences sont les plus marquées, mais ne dépassent pas non plus $8 \%$. Une fois de plus, ce test de validation des données s'avère probant.

Figure 15. Taux de fécondité par âge pour l'arrondissement de Bruxelles selon les recensements de 1981 et de 2001

Générations 1922-1926

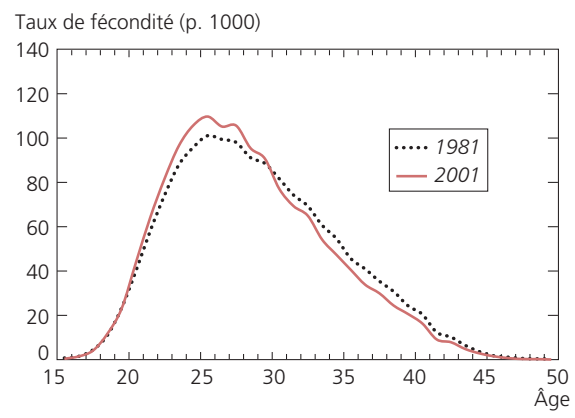

Générations 1932-1936

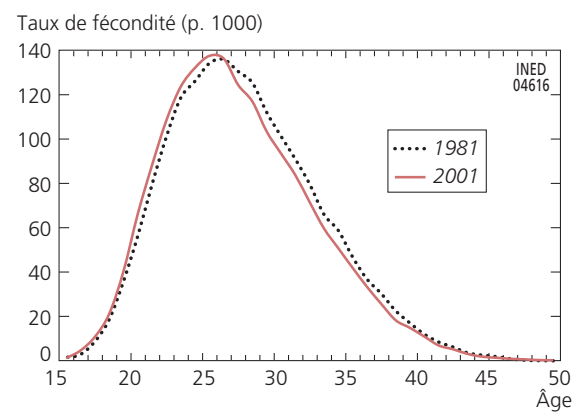

Sources : Recensements de 1981 et de 2001, Belgique.

(35) À ce stade de nos analyses, hormis la moins bonne qualité du recensement de 2001 par rapport à celui de 1981, nous ne pouvons expliquer cet écart par d'éventuels effets de sélection liés à la migration de femmes âgées sans enfant. Quoiqu'il en soit, pour toutes les générations nées avant la Seconde Guerre mondiale, et quelle que soit la source d'observation, Bruxelles se distingue par une très faible fécondité, sensiblement inférieure à la valeur des autres arrondissements. 


\section{À l'échelle des communes en Belgique}

Comme précédemment, le test consiste à confronter, pour les mêmes générations, les descendances finales calculées à partir des recensements de 1981 et de 2001. Il permet de vérifier l'importance des biais de sélection liés aux sorties d'observation par décès et par migration interne et internationale entre 1981 et 2001. Les résultats semblent montrer que, même à l'échelle des communes, là où le poids démographique des migrations est le plus important, les biais de sélection sont modérés (figure 16). Ainsi, pour la génération de femmes nées entre 1922 et 1926, le croisement de leurs descendances finales en 1981 et en 2001, calculées à l'échelle de leur commune de résidence, donne un coefficient de corrélation linéaire de $0,94^{(36)}$ et l'ajustement s'améliore encore pour les générations suivantes.

Figure 16. Comparaison des descendances finales des municipalités aux recensements belges de 1981 et de 2001

pour la génération de femmes nées entre 1922 et 1926

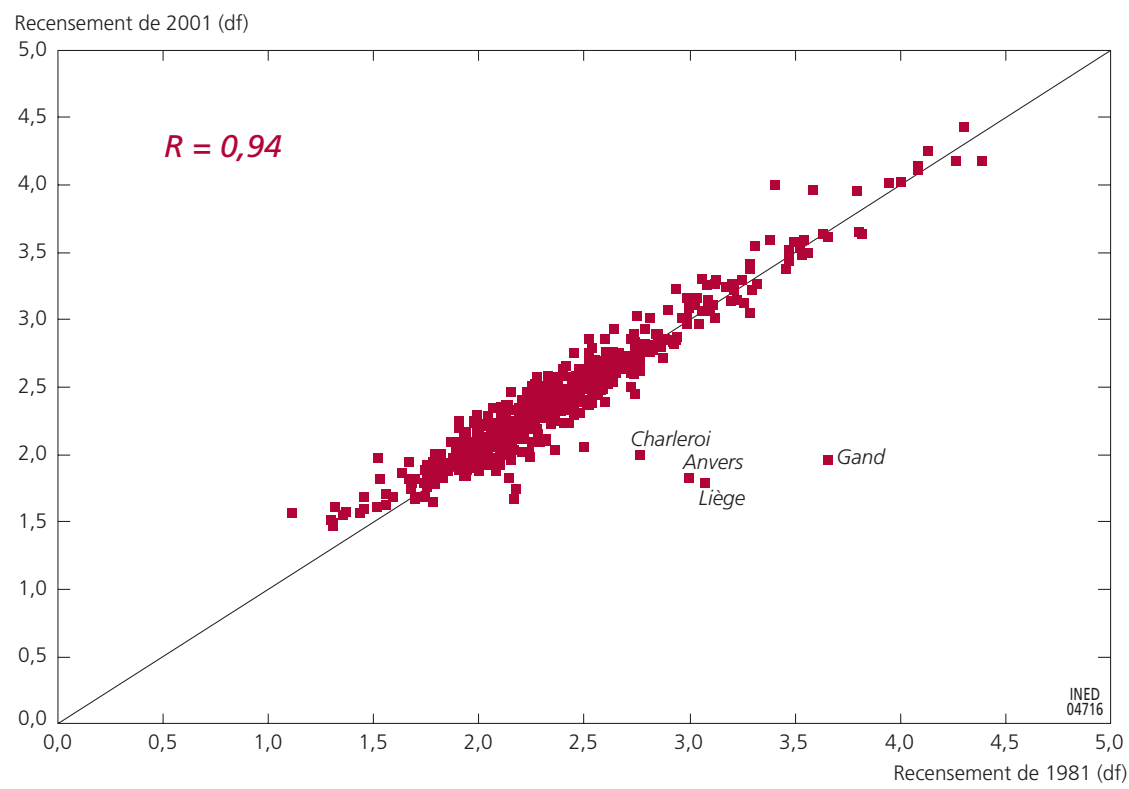

Sources : Recensements de 1981 et de 2001, Belgique.

Prenons le cas de la génération de femmes nées entre 1922 et 1926 . Pour $64 \%$ des communes ( 378 sur 589), la descendance finale présente un écart relatif compris entre $-5 \%$ et $+5 \%$ entre les deux recensements, ce qui correspond au maximum à $\pm 0,1$ enfant pour une descendance finale de 2 enfants. La différence (positive ou négative) varie entre $5 \%$ et $10 \%$ pour $25 \%$ des communes, soit une fluctuation de 0,1 à 0,2 enfant pour une descendance

(36) La même confrontation fournit un coefficient de 0,81 pour les générations nées entre 1917 et 1921. 
similaire. Enfin, pour le reste des communes (10\%), l'écart entre les deux recensements est supérieur à $10 \%$. Parmi celles-ci figurent les grandes villes de Gand, Anvers, Liège et Charleroi, avec des différences importantes, et dans une moindre mesure les communes urbaines de l'agglomération de Bruxelles. Contrairement aux situations nationale et par arrondissement dans lesquelles le recensement de 2001 fournit, pour les mêmes générations, des indices un peu plus élevés que celui de 1981 - ce qui induit une légère sous-fécondité des populations sorties d'observation-, ces communes urbaines présentent des indices de fécondité supérieurs en 1981. Cette situation s'explique probablement par les taux de non-réponse plus élevés lors de l'exercice 2001, en particulier dans les milieux les plus urbanisés ${ }^{(37)}$, plutôt que par une éventuelle surfécondité des populations sorties d'observation, notamment par émigration interne ${ }^{(38)}$. Nous recommandons donc, pour les générations plus anciennes, l'exploitation des données rétrospectives communales extraites du recensement de 1981 plutôt que celles de 2001.

\section{Conclusion}

L'utilisation de données rétrospectives extraites des recensements de la population ou d'enquêtes spécifiques pour reconstituer la fécondité comporte a priori des risques de biais non négligeables. D’une part, des erreurs de mémoire peuvent intervenir dans les réponses, surtout en ce qui concerne les générations de femmes les plus anciennes. D'autre part, on n'observe au recensement qu'une partie des générations concernées. On ne connaît pas la fécondité des femmes sorties d'observation par décès ou par migration, ce qui peut occasionner des biais de sélection. Enfin, une partie de la population ne répond pas à certaines questions et ces individus ne se répartissent pas équitablement sur le territoire ni dans les différents groupes de population (groupes d'âges, groupes sociaux, etc.). Les estimations issues des enquêtes françaises présentent également une certaine variabilité puisqu'on n'observe qu'une part de la population (représentative de la population française) et que les femmes interrogées ne sont pas les mêmes d'une enquête sur l'autre.

Les analyses menées à l'échelle des deux pays montrent que les risques de biais liés aux erreurs de mémoire et aux effets de sélection sont limités et confirment les conclusions d'Andersson et Sobolev (2013) pour les registres de population suédois, et de Neels (2006) et Van Bavel (2014) pour les recensements belges. Les descendances finales des femmes calculées à partir des recensements et des enquêtes s'ajustent remarquablement bien aux descendances

(37) Les taux de non-réponse sont sensiblement plus élevés dans les milieux socialement plus défavorisés et parmi les populations d'origine étrangère, lesquelles se caractérisent par des niveaux de fécondité plus élevés.

(38) Elle ne se vérifie aucunement dans le cadre du processus de périurbanisation que connaissent ces agglomérations urbaines depuis des décennies (Eggerickx et al., 2014). 
finales issues des données de l'état civil ou des résultats obtenus par des méthodes indirectes. De plus, la comparaison de la fécondité des femmes d'une même génération observée à différents recensements ou enquêtes se traduit par des différences minimes, sauf pour les grandes villes.

En théorie, l'un des principaux apports de l'exploitation des données rétrospectives des recensements et dans une moindre mesure des enquêtes, est de pouvoir prendre en considération la dimension spatiale de la fécondité. Dans ce cas, le biais de sélection doit être analysé avec le paramètre supplémentaire des migrations internes. En effet, le lieu de résidence des femmes lors des recensements et des enquêtes ne correspond pas forcement au lieu où leur vie féconde s'est déroulée, et les femmes ont pu migrer entre les recensements. Globalement, les analyses menées ont démontré que ce biais théorique lié aux migrations internes n'invalide pas l'utilisation d'une approche rétrospective et spatiale de la fécondité, même s'il convient de rester prudent dans le cas des grandes villes où les migrations internationales, notamment, peuvent affecter les comparaisons d'un recensement à l'autre.

Une approche rétrospective de la fécondité belge à partir des recensements est donc possible, même à une échelle spatiale fine. Les recensements constituent dès lors une source très riche d'information pour analyser l'évolution de la fécondité au cours du $\mathrm{Xx}^{\mathrm{e}}$ siècle. Ils offrent notamment la possibilité de calculer divers indicateurs de fécondité, comme les descendances finales, les parités, les probabilités d'agrandissement, les intervalles entre naissances, les âges aux maternités, etc. Quant aux enquêtes Famille françaises, même si les variations des résultats sont plus élevées que dans le cas des recensements belges, elles permettent de reconstituer rétrospectivement la fécondité française, y compris au niveau régional, avec un niveau de fiabilité très satisfaisant.

Remerciements : Sandra Brée bénéficie d'une bourse de recherche FSR-COFUND cofinancée par les fonds spéciaux de la recherche de l'Université catholique de Louvain et les actions Marie Curie de la Commission européenne. 


\section{SOURCES STATISTIQUES}

ENQUÊTE FAMILlE, 1954, Insee, Adisp-CMH.

ENQUÊTE FAMILle, 1975, Insee, Adisp -CMH.

ENQUÊTE FAMILLE, 1982, Insee, Adisp -CMH.

ENQUÊTE FAMILLE, 1990, Insee, Adisp -CMH.

ÉTUdE DE L'Histoire FAMiliale, 1999, Insee, Adisp -CMH.

FAMiLle ET LOGEMENTS, 2011, Insee, Adisp -CMH.

RECENSEMENT DE LA POPUlATION ET DES LOGEMENTS 1981, Royaume de Belgique, Direction générale de la Statistique.

ENQUÊTE SOCIOÉCONOMIQUE GÉNÉRALE, 2001, Royaume de Belgique, Direction générale de la Statistique.

\section{RÉFÉRENCES}

ANDERSSON Gunnar, SOBOLEV Boris, 2013, « Small effects of selective migration and selective survival in retrospective studies of fertility ", Revue européenne de démographie, 29(3), p. 345-354.

BACCAÏNI Brigitte, 1993, « Régions attractives et régions répulsives entre 1982 et 1990. Comparaison avec la période 1975-1982 et spécificité des différentes classes d'âges ", Population, 48(6), p. 1791-1811.

BACCAÏNI Brigitte, 1994, "Comportements migratoires et cycles de vie », Espaces, populations, sociétés, 12(1), p. 61-74.

BACCAIINI Brigitte, 2001, « Les migrations internes en France de 1990 à 1999 : l'appel de l'Ouest ", Économie et statistique, n 344(4), p. 39-79.

BACCAÏNI Brigitte, 2007, « Les flux migratoires interrégionaux en France depuis cinquante ans », Population, 62(1), p. 143-160.

BACCAÏNI Brigitte, 2009, « Étudier les migrations résidentielles avec le RP », Document de travail, Insee, $40 \mathrm{p}$.

BACCAÏNI Brigitte, LEVY David, 2009, « Recensement de la population de 2006. Les migrations entre départements : le Sud et l'Ouest toujours très attractifs », Insee première, $\mathrm{n}^{\circ} 1248,4$ p.

BARRE Corinne, VAnderschelden Mélanie, 2004, «Enquête Étude de l'histoire familiale de 1999 », Résultats détaillés, Insee résultats, société, n 33, 47 p. + CD-Rom. 
CASELli Graziella, MESLÉ France, VALLIN Jacques, 1999, « Le triomphe de la médecine », in Bardet J.-P., Dupâquier J. (dir.), Histoires des populations de l'Europe. III. Les temps incertains 1914-1998, Paris, Fayard, p. 125-181.

Costa Rafael, EGGERICKx Thierry, Rizzi Ester, SANDERSON Jean-Paul, 2010, «Analyse spatiale et temporelle de la fécondité en Belgique : une approche communale », Actes du XV Colloque national de démographie (Cudep), La fécondité : représentation, causalité, prospective, Strasbourg.

CRIBIER Françoise, KYCH Alexandre, 1992, « La migration de retraite des Parisiens : une analyse de la propension au départ », Population, 47(3), p. 677-717.

CRIBIER Françoise, KYCH Alexandre, 1993, « Les migrations de retraite des citadins en France », Espace, populations, sociétés, Les migrations de personnes âgées en Europe, 11(3), p. 489-501.

DAGUET Françoise, 2000, «L'évolution de la fécondité des générations nées de 1917 à 1949 : analyse par rang de naissance et niveau de diplôme », Population, 55(6), p. 1021-1034.

DAGUET Françoise, 2002, «Un siècle de fécondité française, caractéristiques et évolution de la fécondité de 1901 à 1999 », Paris, Insee résultats société n 8, 305 p.

DAGUET Françoise, 2005, « Données de démographie régionale, de 1954 à 1999 », Paris, Insee résultats, société, n 49, 48 p. (+ CD Rom).

DAmas Henriette, WATtElaR Christine, Veys Dion, POUlAin Michel (dir.), 1988, La Belgique en 43 arrondissements, Bruxelles, De Boeck Université, 265 p.

DAVIE Emma, MAZUY Magali, 2010, « Fécondité et niveau d'études des femmes en France à partir des enquêtes annuelles de recensement », Population, 65(3), p. 475-512.

DeboOsere Patrick, Demarest Stefan, LORAnT Vincent, MiERMAns Pieter-Jan, PORTET Maria-Isabel, VAN OYEN Herman, 2006, Santé et soins informels, Enquête socio-économique 2001, $\mathrm{n}^{\circ} 1,175 \mathrm{p}$.

DESPLANQUES Guy, 1985, Fécondité générale. Résultats de l'enquête Famille, Paris, Insee, Archives et documents $\mathrm{n}^{\circ} 143,248 \mathrm{p}$.

DESPLANQUES Guy, 1993, «Un premier enfant de plus en plus tard », Paris, Insee première, $\mathrm{n}^{\circ} 247,4$ p.

DESPLANQUES Guy, 1994, «Connaître les migrations », Espace, populations, sociétés, Les migrations internes, 12(1), p. 31-39.

DeSPlANQUES Guy, 2005, «Pour une histoire des enquêtes Famille », in Lefèvre C., Filhon A. (dir.), Histoires de familles, histoires familiales. Les résultats de l'enquête Famille de 1999, Paris, Ined, Cahier n 156, p. 15-27.

DOBLhammer Gabriele, 2000, « Reproductive history and mortality later in life: A comparative study of England and Wales and Austria », Populations Studies, 54(2), p. 169-176.

EGGERICKX Thierry, 2006, « Migrations internationales et populations de nationalité étrangère : quelques aspects démographiques », in Khader B., Martiniello M., Rea A., Timmerman C. (dir.), Penser l'immigration et l'intégration autrement, Initiative belge interuniversitaire sur l'immigration et l'intégration, Bruxelles, Bruylant, p. 29-71.

EGGERICKX Thierry, SANDERSON Jean-Paul, COSTA Rafael, 2014, « La fécondité des migrantes internes en Belgique. Une approche longitudinale et spatiale à partir des données rétrospectives des recensements », Revue Quetelet, $n^{\circ}$ 2(1), p. 39-65.

EGGERICKX Thierry, HeRMiA Jean-Pierre, SURKIJN Johann, WillaERT Didier, 2012, Les migrations internes en Belgique, Monographie 2 de l'Enquête socioéconomique de 2001, Bruxelles, DGSIE, 200 p.

FESTY Patrick, 1979, La fécondité des pays occidentaux de 1870 à 1970, Paris, Ined, Cahier $\mathrm{n}^{\circ} 85,400 \mathrm{p}$. 
HÉRAN François, 2005, Préface « Du public, du privé et de l'intime dans l'enquête Famille 1999. Témoignage d'un concepteur sur une enquête qui revient de loin », in Lefèvre C., Filhon A. (dir.), Histoires de familles, histoires familiales. Les résultats de l'enquête Famille de 1999, Paris, Ined, Cahier n 156, p. 15-27.

INSEE, 1982, Description du fichier codifié, enquête 1982, 408 p.

INSEE, 1990, Description du fichier codifié, enquête 1990, 289 p.

INSEE, 2002, Étude de l'histoire familiale en 1999, Documentation des fichiers, 214 p.

INSEE, 2014, Enquête Famille et logements, Dictionnaire des codes, 229 p.

LAVERTU Jacques, 1997, «Fécondité et calendrier de constitution des familles : enquête Famille 1990 », Paris, Insee résultats, Démographie-société, n 579, 144 p.

LE BRAS Hervé, 1995, Les trois France, Paris, Odile Jacob (lère édition : 1986), 476 p.

LesthaEgHE Ron, 1977, The Decline of Belgian Fertility, 1800-1970, Princeton University Press, $350 \mathrm{p}$.

LODEWIJCKX Edith, 1999, «Fertility and family surveys in countries of the ECE region, standard country report, Belgium », Economic Studies, $n^{\circ} 10$, Nations unies, New-York/Genève, 96 p.

MASUY-STROOBANT Godelieve, 1976, « Qui se rassemble, s'assemble. I - Les dimensions de la fécondité des arrondissements belges, comparaison 1961-1970 », Population et famille, 1(37), p. 1-36.

MASUY-STROOBAnT Godelieve, 1977, « Qui se rassemble, s'assemble. II - Une typologie de la fécondité générale des arrondissements belges, comparaison 1961-1970 », Population et famille, 3(42), p. 113-133.

Mazuy Magali, Toulemon Laurent, 2013, « Fécondité des hommes et des femmes selon le niveau d'études d'après l'enquête EFL », Programme « des lieux aux liens » (LiLi), Groupe d'exploitation de l'enquête Famille et logements (EFL), Ined, 23 septembre 2013.

NATIONS UNIES, 1958, « Recent trends in fertility in industrialized countries », Population Studies, $\mathrm{n}^{\circ} 27,182 \mathrm{p}$.

NEELS Karel, 2006, Reproductive Strategies in Belgian Fertility, 1960-1990, Centrum voor Bevolkings- en Gezinsstudie, Brussel, Den Haag, 314 p.

NEELS Karel, 2010, « Period and cohort effects in Belgian nuptiality and fertility: 1960-1990 », in Eggerickx T., Sanderson J.-P. (dir.), Histoire de la population de la Belgique et de ses territoires, Chaire Que telet 2005, Centre de recherche en démographie et sociétés, Presses universitaires de Louvain, Louvain-la-Neuve, p. $463-488$.

ORIS Michel, 2009, «Aux origines de la deuxième transition démographique en Europe : une relecture des enquêtes fécondité-famille entre 1966 et 1972 », in Marcoux R. (dir.), Mémoires et démographie, aspects croisés au Sud et au Nord, Québec, Presses universitaires de Laval, Cahiers du Cieq, p. 33-44.

SARDON Jean-Paul, 1990a, « Le remplacement des générations en Europe depuis le début du siècle », Population, 45(6), p. 947-968.

SARDON Jean-Paul, 1990b, "La fécondité des cohortes dans les États membres du Conseil de l'Europe », Conseil de l'Europe, Études démographiques, n² 21, 205 p.

SCHOENMAECKERS Ronald, LODEWIJCKX Edith, GADEYNE Sylvie, 2002, Nuptialité et fécondité. Partie B : fécondité, Recensement général de la population et des logements au $1^{\text {er }}$ mars 1991, Bruxelles, INS, SSTC, 131 p. + annexes.

TODD Emmanuel, LE BRAS Hervé, 2014, L'invention de la France, Paris, Gallimard

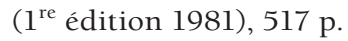

TOULEMON Laurent, 1995, « Très peu de couples restent volontairement sans enfant », Population, 50(4-5), p. 1079-1109. 
TOUlEMON Laurent, 2001, « Combien d'enfants, combien de frères et sœurs depuis cent ans? ", Population et sociétés, n० 374,4 p.

VAN BAVEL Jan, 2014, «The mid-twentieth century Baby Boom and the changing educational gradient in Belgian cohort fertility », Demographic Research, 30(33), p. 925-962.

WUNSCH Guillaume, 2001, «L'analyse de la fécondité par cohorte », in Caselli G., Vallin J., Wunsch G. (dir.), Démographie : analyse et synthèse. I. La dynamique des populations, Paris, Ined, p. 149-163. 


\section{Sandra BrÉE, Thierry EgGERICKX, Jean-Paul SANDERSON, Rafael COSTA • COMPARAISON DES DONNÉES RÉTROSPECTIVES DE FÉCONDITÉ DANS LES RECENSEMENTS EN BELGIQUE ET LES ENQUÊTES FAMILLE EN FRANCE}

Les comportements de fécondité au cours de la première moitié du $\mathrm{XX}^{\mathrm{e}}$ siècle restent relativement méconnus, en raison notamment des législations interdisant l'exploitation de données individuelles vieilles de moins de 100 ans, ou de la pauvreté relative des données agrégées transversales. L'objectif de cet article est de vérifier s'il est possible de combler cette lacune en exploitant de manière rétrospective les questions posées sur la vie féconde des femmes lors des enquêtes Famille menées en France et des recensements de la population en Belgique. Les analyses ont démontré que les risques de biais liés aux erreurs de mémoire, aux effets de sélection et aux non-réponses, inhérents à ces observations rétrospectives, sont limités. La reconstitution de la vie génésique des différentes générations de femmes interrogées au moment de l'enquête ou du recensement offre donc un potentiel analytique important. Elle permet de calculer des indices plus fins et diversifiés de l'intensité et du calendrier de la fécondité pour des générations de femmes nées à partir des dernières décennies du XIX ${ }^{\mathrm{e}}$ siècle. Cela permet par ailleurs de prendre en considération la dimension spatiale de la fécondité, à l'échelle des régions en France et à celle des arrondissements et même des communes en Belgique.

\section{Sandra BRÉE, Thierry EgGeriCKX, Jean-Paul SANDERSON, Rafael COSTA • COMPARISON of Retrospective fertility Data from Censuses in Belgium and family SuRveys in FRANCE}

Fertility behaviour in France and Belgium in the first half of the twentieth century is still quite poorly understood, owing mainly to legislation that prohibits the use of individual data less than 100 years old, and to the paucity of cross-sectional aggregate data. This article tests whether it is possible to bridge that gap via a retrospective approach, using the questions about women's reproductive life from family surveys conducted in France and population censuses in Belgium. The analyses show that the risks of bias related to recall errors, selection effects and non-response, inherent in these retrospective observations, are limited. The reconstituted birth histories of the various cohorts of women recorded at the time of the survey or census therefore offer considerable research potential, making it possible to calculate more detailed, more diversified indicators of the intensity and timing of fertility for cohorts of women born since the end of the nineteenth century. They also enable us to capture the spatial dimension of fertility, at the level of the regions in France, and of the arrondissements and even the municipalities in Belgium.

\section{Sandra Brée, Thierry EgGERICKX, Jean-Paul SANDERSON, Rafael Costa • COMPARACIÓN DE LOS DATOS RETROSPECTIVOS DE FECUNDIDAD EN LOS CENSOS EN BÉLGICA Y EN LAS ENCUESTAS FAMILIA, EN FRANCIA}

Los comportamientos de fecundidad durante la primera mitad del siglo XX son relativamente poco conocidos, a causa sobre todo de las legislaciones que prohíben la explotación de datos individuales de menos de 100 años o de la relativa pobreza de los datos agregados transversales. El objetivo de este artículo es comprobar si se puede rellenar este vacío explotando retrospectivamente las respuestas a las preguntas sobre la vida fecunda de las mujeres, hechas en las encuestas Familia francesas y en los censos belgas. Los análisis han demostrado que los riesgos de sesgo asociados a los errores de memoria, a los efectos de selección y a las no-respuestas, inherentes a este tipo de observación, son limitados. La reconstitución de la vida genésica de las diferentes generaciones de mujeres interrogadas en la encuesta o en el censo, ofrece así un alto potencial analítico. Es posible, en efecto, calcular índices más detallados y diversificados de la intensidad y del calendario de la fecundidad en las generaciones nacidas en las últimas décadas del siglo XIX. Dicha reconstitución permite también considerar la dimensión espacial de la fecundidad, a nivel regional en Francia y a nivel de distrito e incluso de municipio en Bélgica.

Mots-clés : Fécondité, données rétrospectives, enquête, recensement, France, Belgique.

Keywords: Fertility, retrospective data, survey, census, France, Belgium. 